\title{
Research Paper \\ The Impact of International Oil Prices on the Return of the Market Portfolio - A Study of the Iraq Stock Exchange for the Period 2012-2019
}

Journal of

\section{TANMIYAT AL- RAFIDAIN}

\section{(TANRA)}

A scientific, quarterly, international, open access, and peer-reviewed journal

Vol. 40, No. 132

Dec. 2021

(C) University of Mosul | College of Administration and Economics, Mosul, Iraq.

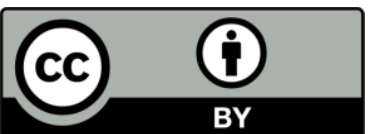

TANRA retains the copyright of published articles, which is released under a "Creative Commons Attribution License for CC-BY-4.0" enabling the unrestricted use, distribution, and reproduction of an article in any medium, provided that the original work is properly cited.

Citation: AL-Mizori, Ashti A. A., Mohammad, Ahmed J.(2021).'The Impact of International Oil Prices on the Return of the Market Portfolio - A Study of the Iraq Stock Exchange for the Period 2012-2019". TANMIYAT ALRAFIDAIN, 40 (132), 225-39, https://doi.org/ 10.33899/tanra.2021.130175.10 94

P-ISSN: 1609-591X e-ISSN: 2664-276X tanmiyat.mosuljournals.com

\section{Ashti A. A. AL-Mizori ${ }^{1}$; Ahmed J. Mohammad ${ }^{2}$}

Financial and Banking SciencesCollege of Administration and EconomicsUniversity of Mosul ${ }^{1}$

Financial and Banking SciencesCollege of Administration and EconomicsUniversity of Basra ${ }^{2}$

Corresponding author: Ashti A. A. AL-Mizori, Financial and Banking SciencesCollege of Administration and Economics-University of Mosul

Ashti800@gmail.com

DOI: https://doi.org/ 10.33899/tanra.2021.130175.1094

Article History: Received:19/5/2021; Revised: 2/6/2021; Accepted: 9/6/2021; Published: 1/12/2021.

\section{Abstract}

This study dealt with the issue of the investment portfolio through some objectives, the most important of which is about the impact of oil price fluctuations, along with the main economic variables, on the returns of the market portfolio, by applying to all sectors listed in the Iraqi Stock Exchange, based on the quarterly relative data with 32 views during the period from 2012-2019, using many different regression analysis models as well as programs and mathematical-statistical methods. To that, the study reached some conclusions, the most important of which is that there is an indirect relationship between oil price rates and economic variables with rates of returns for the market portfolio, and this relationship is based on a variable There is only one broker, which is the money supply, and it is also possible to build several models that explain at high rates between the changes in the rates of oil prices with the rates of returns of the Iraq Stock Exchange portfolio, and the study recommends the necessity of diversification in the investment portfolio by choosing the economic sectors that are affected by varying levels with changes Oil prices to achieve a kind of balance between the return and risk of the market portfolio.

\section{Keywords:}

Oil Prices, Economic Variables, Market Portfolio. 


\section{ورقة بحثية

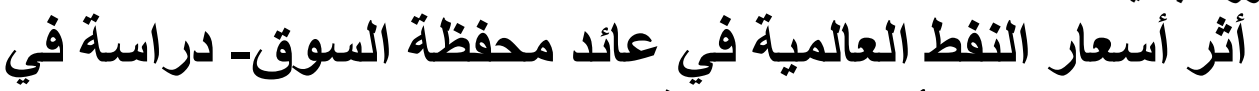

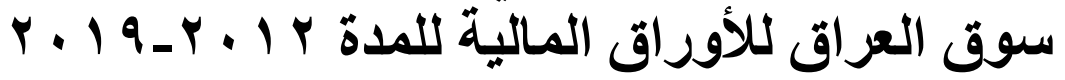

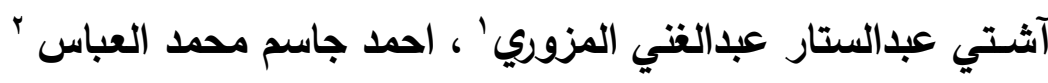

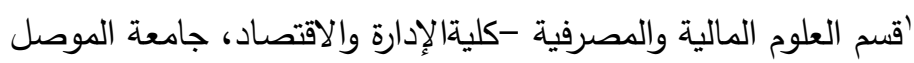
كَّم العلوم المالية والمصرفية -كليةالإدارة والاقتصاد، جامعة البصرة.

المؤلف المراسل: آشتي عبدالستار عبدالغني المزوري - قسم العلوم المالية والمصرفية ، كلية الإدارة

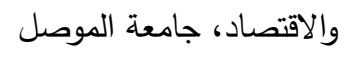

\section{Ashti800@gmail.com}

DOI: https://doi.org/ 10.33899/tanra.2021.130175.1094

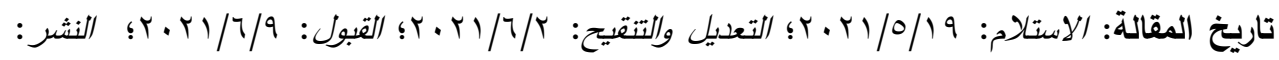

$$
. r \cdot T / / T / 1
$$

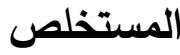

تناولت هذه الدراسة موضوع المحفظة الاستثمارية من خلال جملة من الأهداف /همها عن بيان مدى تأثير

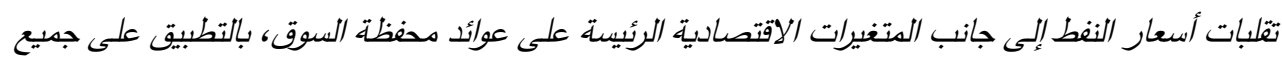

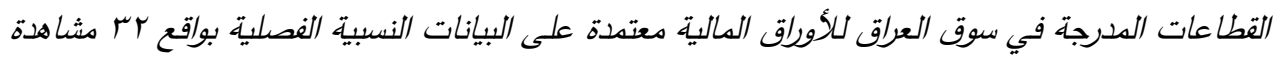

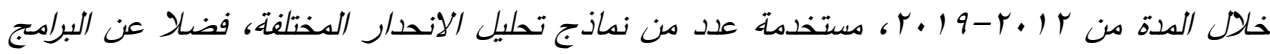

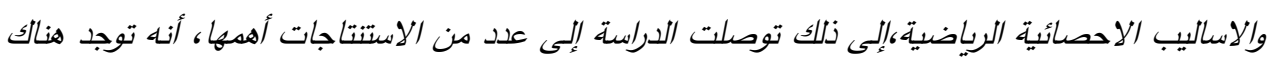

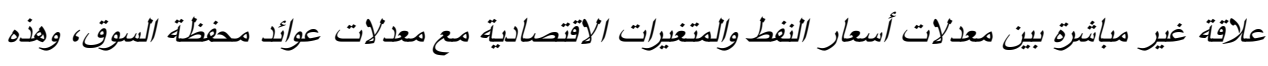

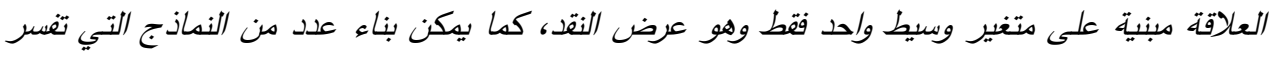

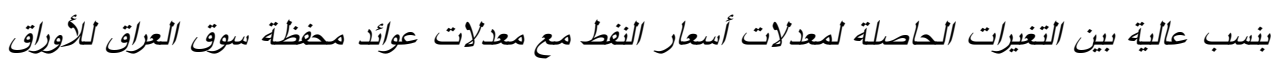

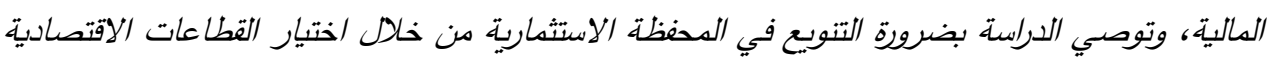

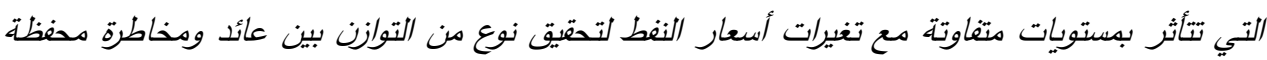
السوق.

\section{الكلمات الرئيسة}

أسعار النفط، متغيرات اقتصادية، محفظة السوق.

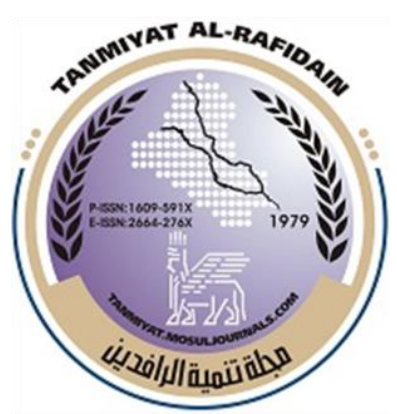

هبلة

\section{تقمهية الرافدبن}

(TANRA) دولية، مفتوحة الوصول، محكمة.

$$
\begin{aligned}
& \text { المجلد (••()، العدد (Y (I))؛ } \\
& \text { كانون الثاني r.r. }
\end{aligned}
$$

ج جامعة الموصل | كلية الإدارة والاقتصاد، الدوصل، العراق.

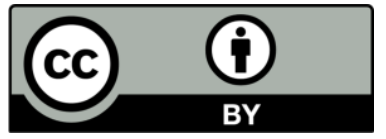

تحتف (TANRA) بحقوق الطبع والنشر للمقالات المنشورة، والتي يتم إصدارها بموجب ترخيص

\lrcorner (Creative Commons Attribution) (CC-BY-4.0)

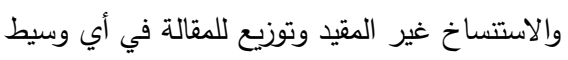
نقل، بشرط اقتباس العمل الأصلي بثكل صحيح.

الاقتباس: المزوري، آثنتي عبدالستار

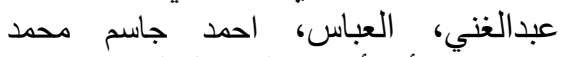

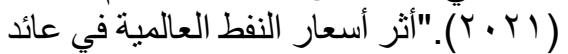

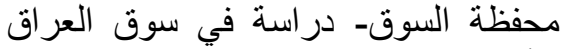

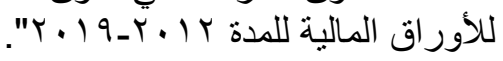

تنمية/الرافدين، • ؛ ( I ) )، آ-، https://doi.org/

10.33899/tanra.2021.130175.10 94

P-ISSN: 1609-591X

e-ISSN: 2664-276X

tanmiyat.mosuljournals.com 
لمعدمة

يُشكل مطلع القرن العشرين البدايات الأولى لتربع النفط على قمة هرم السلع الاستراتيجية العالمية سواء

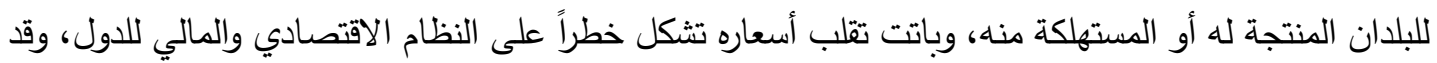
يؤدي ذلك بالدور المحوري والفاعل في التأثير على معظم المتغيرات الاقتصادية والمالية للدولة، ومنها عوائد النشاط الاستثماري في الأسواق المالية، وهو ما اهتمت به وفسرته الأطر النظرية والفكرية في أدبيات الإدارة

وفي خضم الاهتمام بهذا التأثير أثارت الدراسات الاقتصادية والمالية إلى أنه في ظل التقلبات المستمرة لأسعار النفط، فإنه ينبغي على المستثمرين في الأسواق المالية بالسعي إلى تعظيم المنافع لأوراقهم المالية عبر

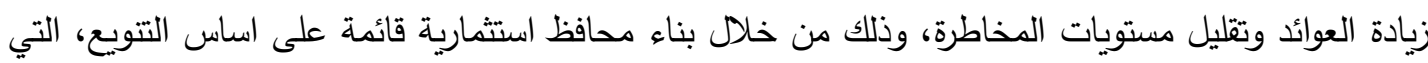
تعد من الأدوات الاستثمارية الحديثة في البيئة الاستثمارية. مشكلة البحث يعد الافتقار إلى سبل تثكيل محافظ استثمارية كفوة، ومحاولة تحقيق التوازن المطلوب بين تعظيم عوائد المحفظة الاستثمارية وتقليل مخاطرها في آن واحد، هو من أحد أعقد الاشكاليات التي تواجه المستثرين في لي أسواق المال، لاسيما في المشروعات العاملة ضمن بيئة الاقتصاديات الريعية، الذي يخضع لحالات عدم استقرارية ايراداتها النفطية ومن بينها بلد الدراسة، وتتمثل مشكلة البحث في التساؤل الآتي:

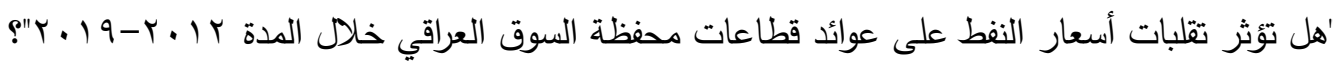
أهمية البحث تكتسب اهمية البحث بتسليط الضوه على مناقثة الموضوع رغم تتاولها من قبل دراسات أخرى، الا ان

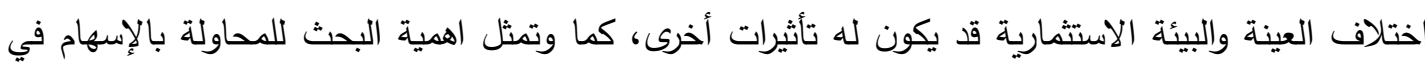

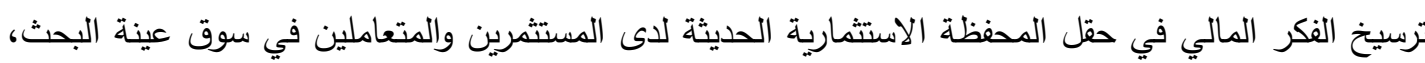
كونه يعد أحد اهم الحقول المعرفية المعاصرة في مجال إدارة المال والاستثمار . فرضية البحث يفترض البحث أنه توجد علاقة تأثير ضعيفة بين تقلبات أسعار النفط وعوائد جميع قطاعات محفظة السوق

$$
\text { هال العراقي. }
$$

"يهدف البحث إلى بيان علاقة تأثير تقلبات أسعار النفط على عوائد محفظة سوق العراق للأوراق المالية

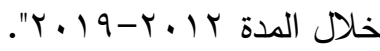
منهج البحث تم الاعتماد على المنهج التحليلي من خلال استخدام عدد من نماذج الانحدار المختلفة وأهمها انحدار متعدد الحدود، والتي تربط بين متغيرات الدراسة من المتغير المستقل المتمثل بأسعار النفط، والمتغيرات الوسيطة المتمثلة 
بكل من (الناتج المحلي الاجمالي، عرض النقد، سعر الفائدة، معدل التضخم، وسعر الصرف)، ومع المتغير

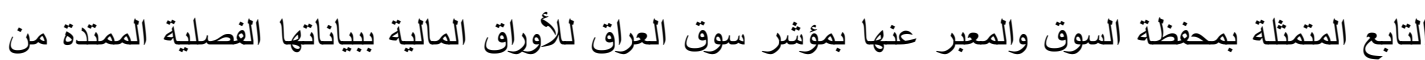

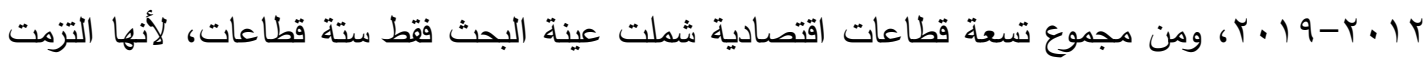
بنشر بياناتها الفصلية خلال مدة البحث. 1. أنظية معرفية لمتغيرات البحث

1- 1 ـ أثر تقلبات أسعار النفط على متغيرات الاقتصاد الكلي لفئ قد يأتي المفهوم الدقيق التقلبات سعر النفط بأنها تلك المشاهد التي تكون فيها عدم استقرارية وبصورة متكررة في أسواق النفط العالمية، متمثلة بالارتفاعات والانخفاضات الكبيرة والمتعاقبة التي تطرأ على سعر برميل النفط

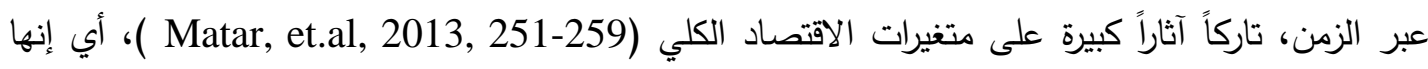
تمارس تأثيراً على النشاط الاقتصادي، وذلك عبر العديد من قنوات متغيرات الاقتصاد الكلي والأساسية منها

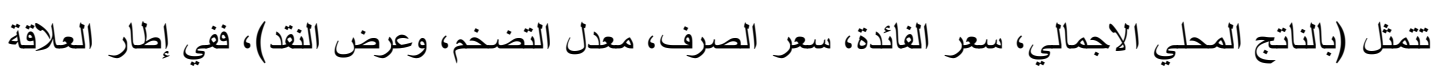

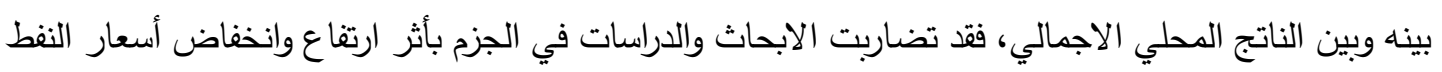
للدول المصدرة والمستوردة للنفط، إذ أثار فريق منهم بوجود علاقة موجبة وسالبة، وادعى الفريق الآخر بعلاقة الإنة

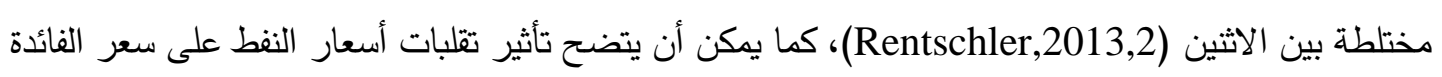

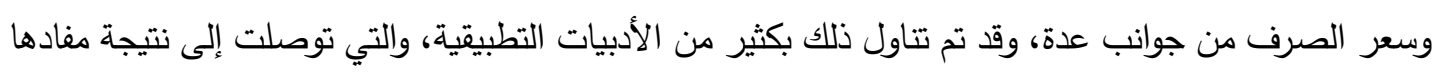

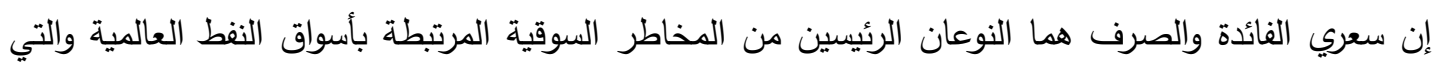

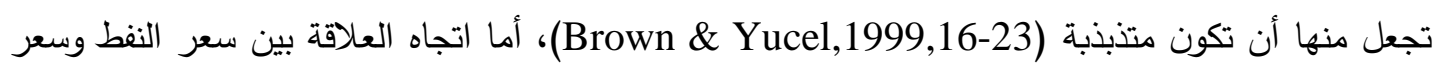

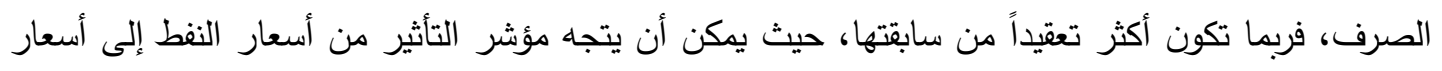

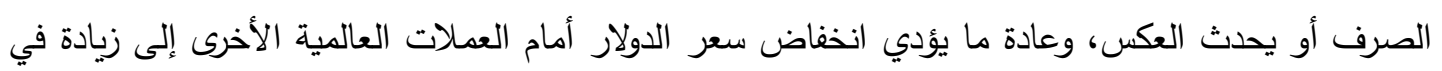

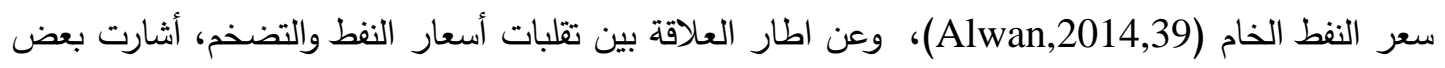

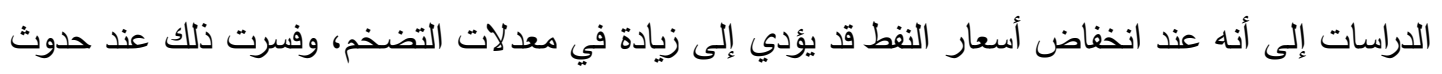

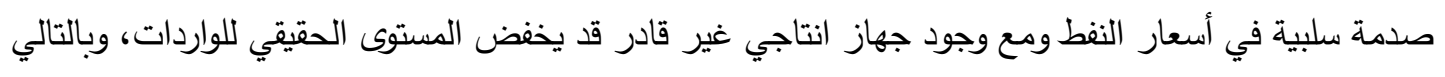

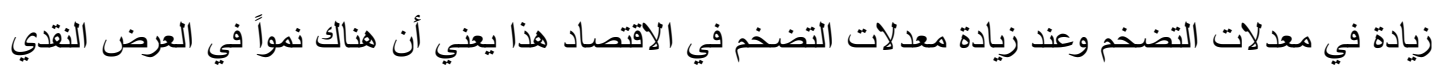

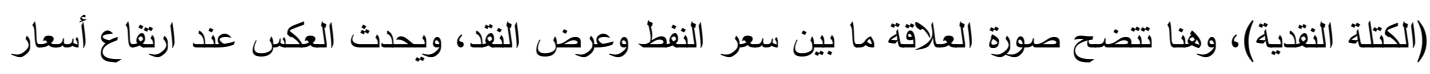
النفط (Aziza, et.al,2019,2-14) (Ito,2008,3-9)، يلمح مدا ذُكر آنفاً أن تكرار عدم استقرارية أسعار النفط الخام، قد تترك من ورائها انعكاسات متباينة على مختلف متغيرات الاقتصاد الكلي لا سيما الاساسية منها،

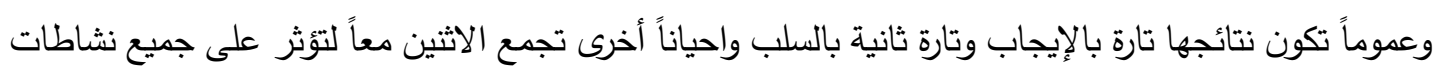

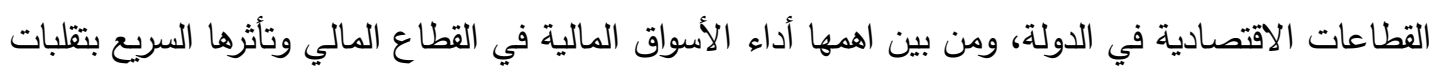

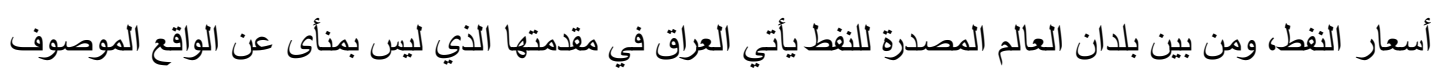

اعلاه. 


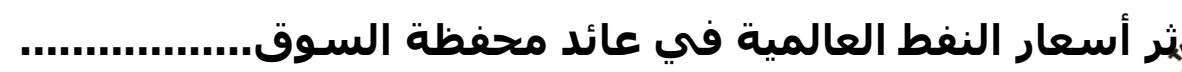

ץ-1 . تأثير عوامل متغيرات الاقتصاد الكلي على مكونات المحفظة الاستثمارية

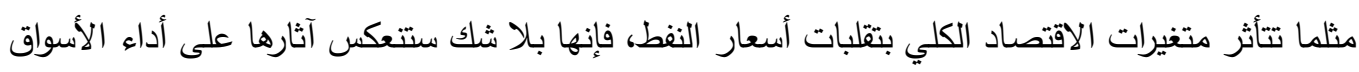

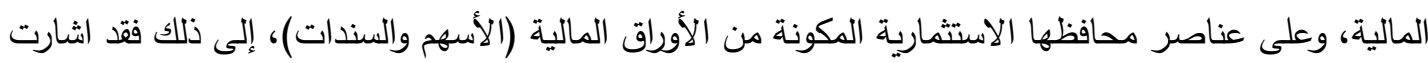

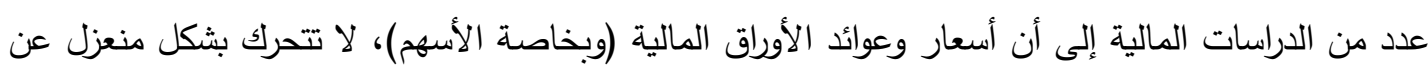

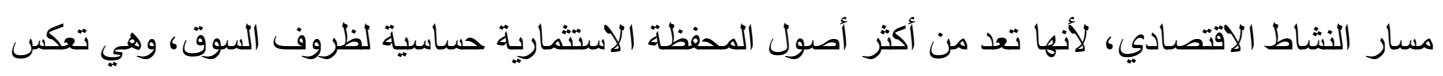

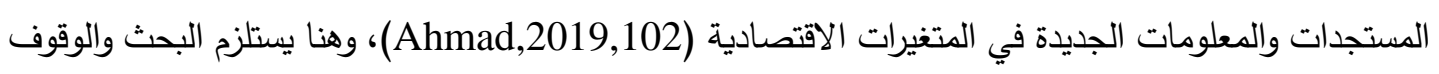

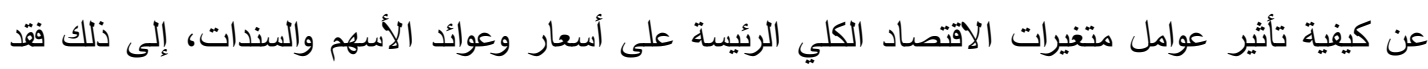

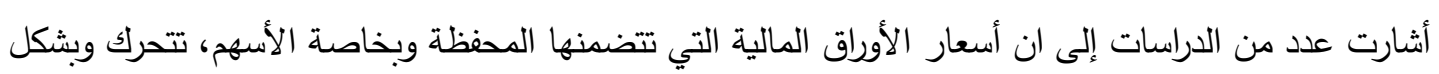

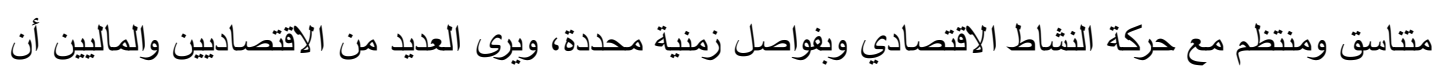

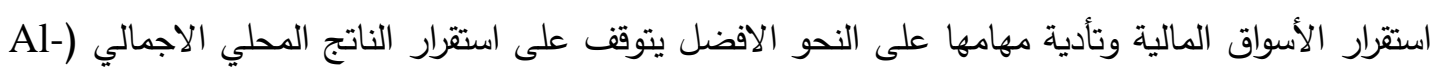

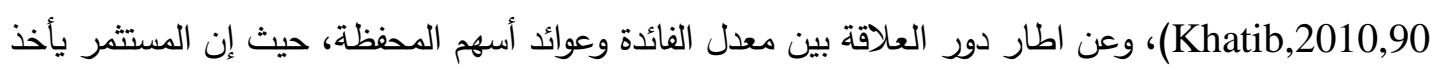
بعين الاعتبار مستوى الفائدة التي تتسم بنسبة قليلة من المخاطرة، وعلى ضوء دذان هذا السعر يكون الاستثمار في

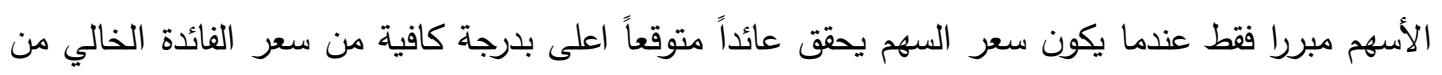

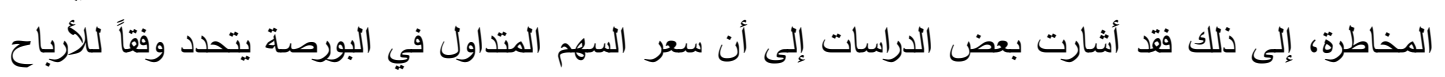

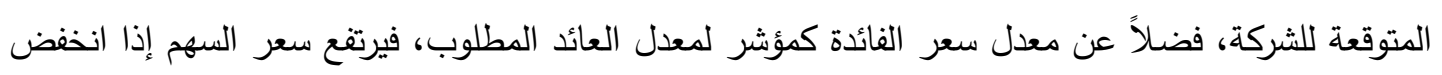

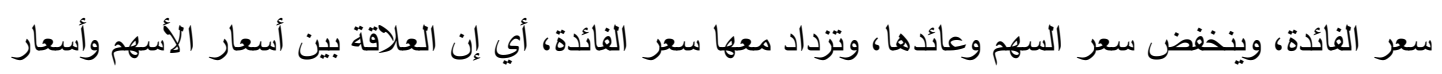
الفائدة علاقة عكسية (Maysami,et.al,2004,48-61)، وعن تأثير سعر الصرف على عوائد المحفظة، ففي

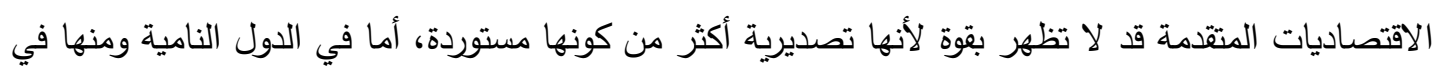
الاقتصاديات العببية فتأثير سعر الصرف يكون من خلال تأثيره على التدفقات النقدية للمشاريع المستوردة، واستبدال العملة الوطنية بالعملة المحلية في أوقات ضعف العملة المحلية يؤدي إلى انخفاض أسعار الأسهم، وقد

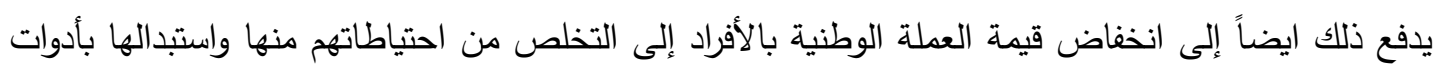

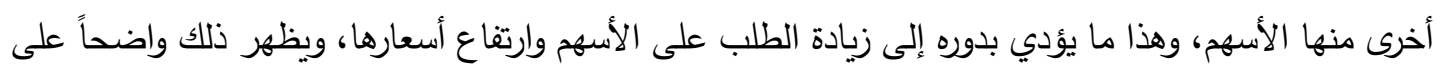

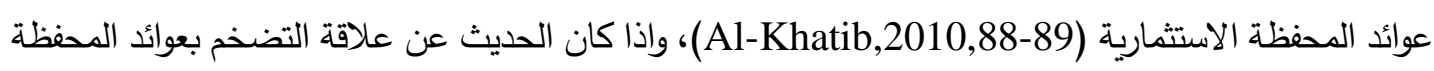
فإنه عند ارتفاع معدلات التضخم، ستتخفض القوة الثرائية للنقود، وبالتالي تتخفض التخان القيمة المتوقعة للتدفقات النقدية المستقبلية للاستثمارات، وفي هذه الحالة يقوم المستثرون بمحاولات بيع أسهم محافظهم، وهذا ما سيؤدي

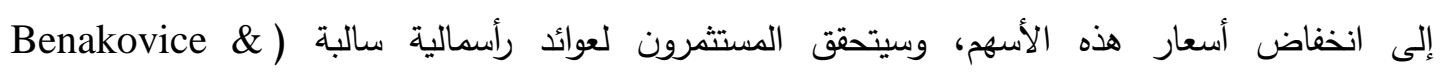

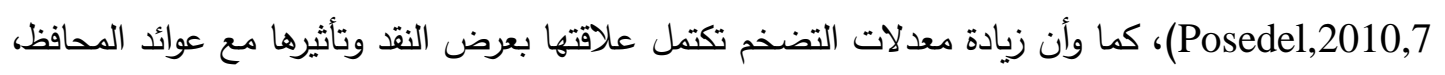

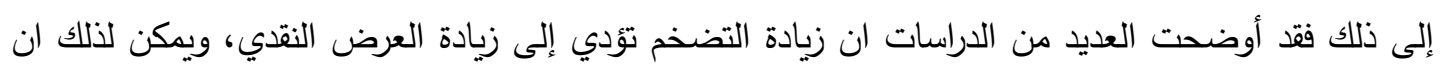

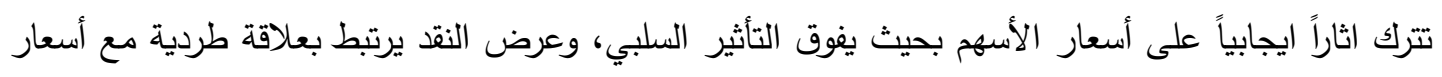
الأسهم وهو ما توصل إليه (Friedman)، والعكس يصبح صحيحاً اذا ما اتضح ان لزيادة عرض التهار النقد أثراً سلبياً 
على عوائد المحفظة، بحيث يفوق الأثر الايجابي في النشاط الاقتصادي ومن ثم في أسعار أسهم المحفظة

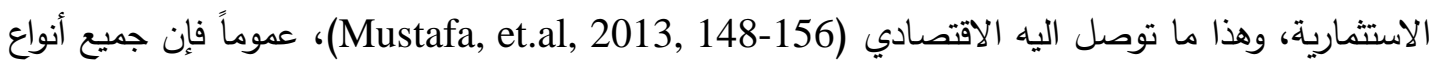
متغيرات الاقتصاد الكلي آنفة الذكر ، تتأثر بتقلبات أسعار النفط لاسيما في الاقتصاديات النفطية، وتتعكس آثارها

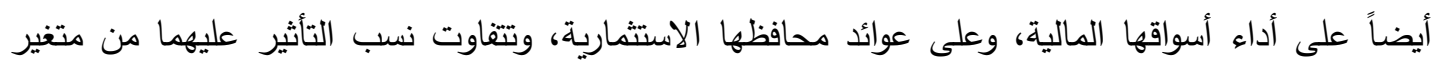
اقتصادي إلى آخر .

r-1 ـ مؤشر السوق المالي أداة لبناء المحفظة الاستثمارية في ظل التقلبات الاقتصادية

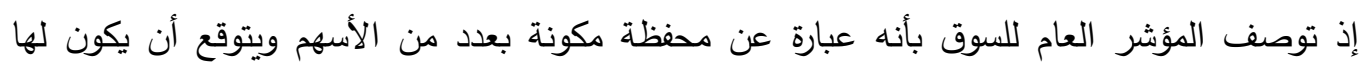

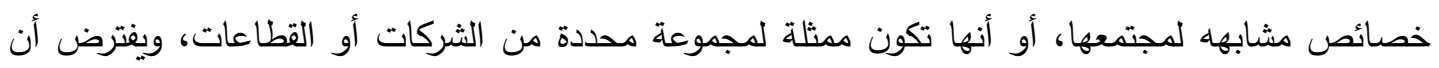
يتعقب المؤشر أداء تلك الثركات او القطاعات (Al-Mansour,2018,46)، إلى ذلك ذهب كل ( Clare \&

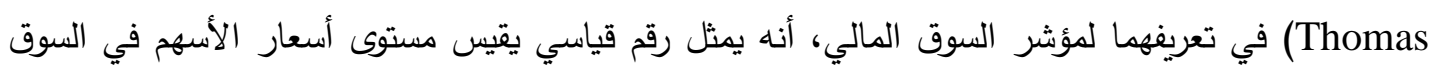

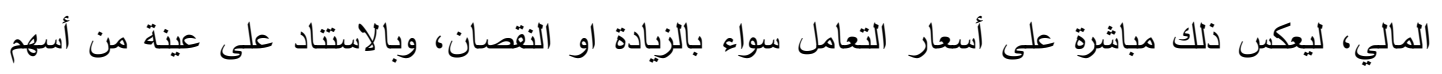

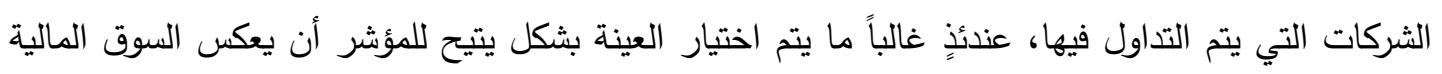
المراد قياسها (Clare \& Thomas, 2015,4)، إلى ذلك فقد تباينت وجهات نظر الباحثين حول تبرير استخدام

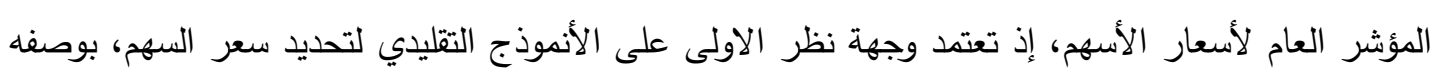

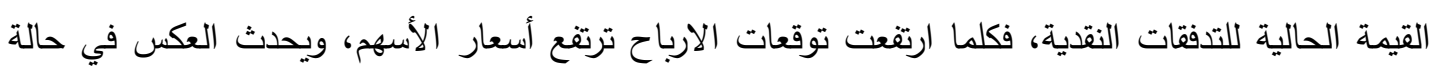
الانخفاض (Al-Shakarji \& Al-Taj al-Din,2008,75-76)، كما واثبتت العديد من الدراسات التطبيقية، توفئ، أنه بإمكان المؤشرات المالية ان تسهم في التعرف على عوامل متغيرات الاقتصادي الكلي، وتؤثر ذلك على الكي

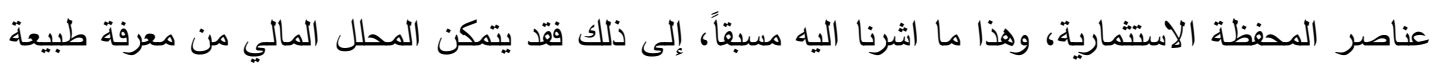

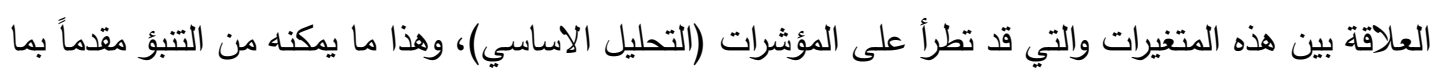

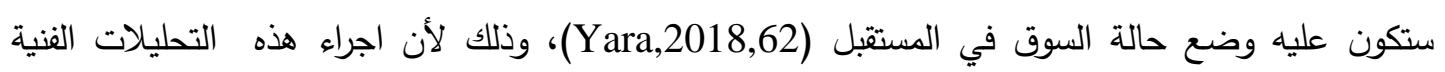

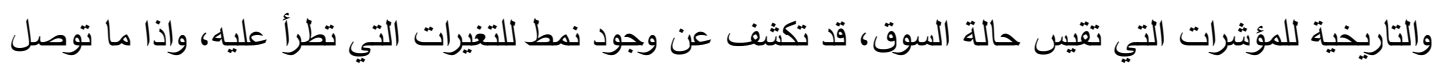

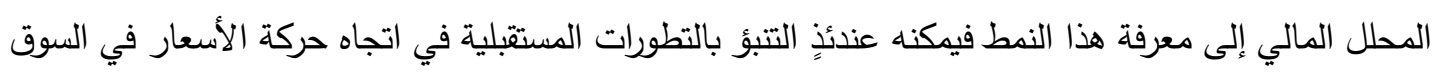
المالية (The Arab Planning Institute,2004,7)، يخلص مما سبق أن مؤشرات الأسواق المالية، فضلاً عن كونها أداة مهمة لمعرفة الأداء الاقتصادي في الدولة أو للتنبؤ بما سيكون الوضع عليه في المستقبل، فإنها

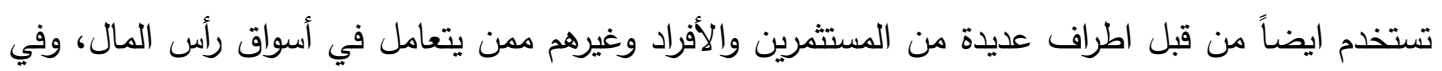
طليعة تلك الاستخدامات أنها تعطي فكرة سريعة للعوائد المتولدة عن المحفظة الاستثمارية للمستثمرين، والحكم على مستوى أداء المديرين المحترفين القائمين على إدارة محافظ الاستثمار في المؤسسات المتخصصة بعدئ بعمليات

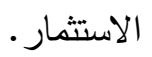




\section{r - تفية تحليلية لمتغيرات البحث}

1-r ب . توصيف المتغيرات المستخدمة في نماذج البحث

قبل الخوض في تفاصيل توصيف متغيرات البحث، لابد من الاشارة إلى البيانات التي اعتمد في البحث، لوني،

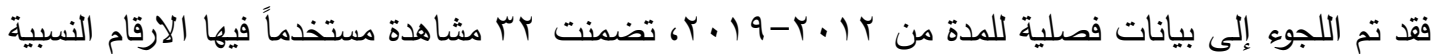
لجميع متغيرات البحث، وقد تم الاعتماد على البيانات المتوفرة على المواقع الالكترونية للبنك الدولي والبنك

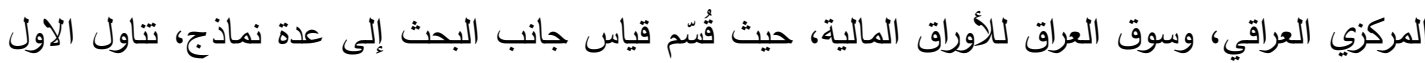

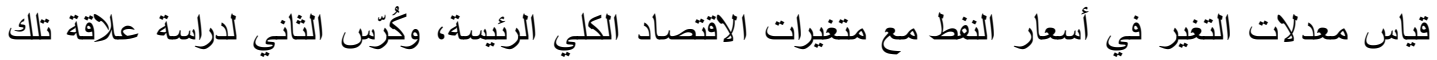
المتغيرات مع معدل عائد محفظة السوق، ومن ثم بيان آثارها على مكونات عوائد محفظة السوق ممثلة بقطاعاتها لهاتيا الاقتصادية كل على حدة، ولغرض توضيح هيكل الأنموذج القياسي المستخدم للبحث، فإنه تم تحديد المتغيرات

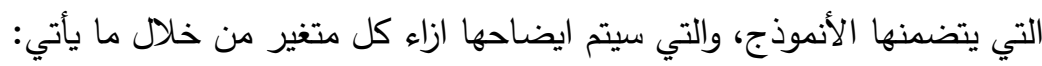
أ- المتغير المستقل: ورمز له بـ X) وعبر عنه بمعدلات أسعار النفط العالمية، وتم الاعتماد على السعر لئير المعدل العالمي لأسعار النفط الثلاثة الرئيسة في العالم. ب- المتغيرات الوسيطة: وشملت خمسة متغيرات وعلى النحو الآتي:

1- الناتج المحلي الاجمالي: رمز له بـ (Z1) معبراً عنه بالنشاط الاقتصادي الإجمالي، وبالأسعار الجارية. r- عرض النقد: رمز لله بـ (Z2) وتضمن ذلك بالمفهوم الضيق (M1) معتمدة على بيانات تم استخراجها من

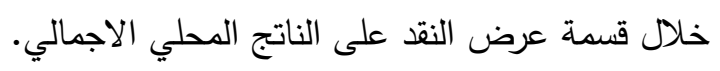
r- سعر الفائدة: رمز له بـ (Z3) وتم الاعتماد على أسعار الفائدة لأذونات الخزينة.

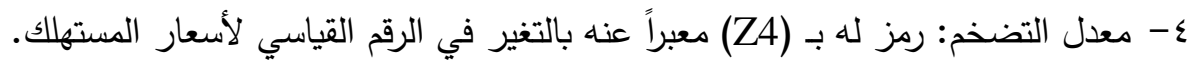

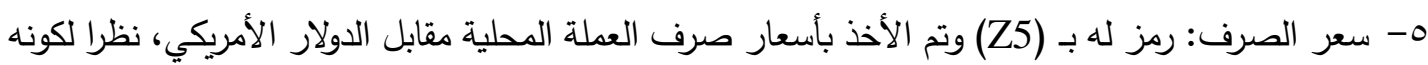
من أهم العملات المطلوبة والمتداولة على نطاق واسع في العراق. ت- المتغير التابع: رمز له بـ (Y) وتم تحديد هذا المتغير من خلال المؤشر العام للسوق المالي ممثلة بعائد

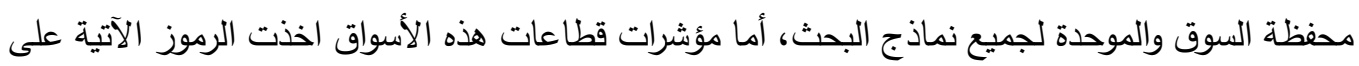

$$
\text { التوالي (Y, (Y) }
$$

وبعد تحديد المتغيرات المستقلة والوسيطة والتابعة المذكورة آنفاً، يمكن صياغة العلاقة الدالية لمتغيرات الأنموذج والتي تأخذ الثكل الآتي:

$$
\begin{aligned}
& Y_{t}=\alpha_{0}+\beta_{1} Z_{1 t}+\beta_{2} Z_{2 t}+\beta_{3} Z_{3 t}+\beta_{4} Z_{4 t}+\beta_{5} Z_{5 t}+\varepsilon_{0 t} \ldots \ldots \ldots \\
& \text { حيث إن: }
\end{aligned}
$$$$
\ldots \ldots Z_{i t}=\alpha_{i}+y_{i} X_{t}+\varepsilon_{i t} ; \text { where } t=1,2 \ldots, N ; i=1,2, \ldots, 5
$$

Y

X 
ط: المتغير الوسيط í، وهو يمثل كل من الناتج المحلي الاجمالي وعرض النقد وسعر الفائدة ومعدل

$$
\text { التضخم وسعر الصرف على التوالي. }
$$

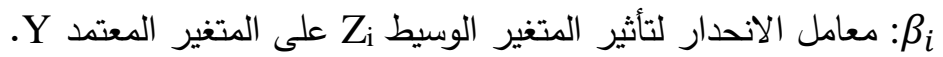

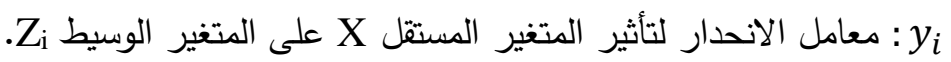
ع

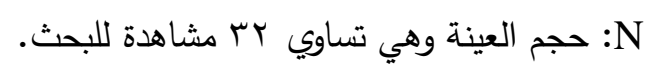

والأنموذج الرياضي لهاتين المعادلتين اعلاه تعبر عن أسعار النفط ومحفظة السوق، وهو أنموذج خطي، بمعنى أن العلاقة بين المتغيرات هي علاقة خطية، وتعتبر المتغيرات الوسيطة متغيرات مستقلة في المعادلة الاولى، ومتغيرات معتمدة في المعادلة الثانية. r-r. r. العلاقة بين متغيرات البحث

من المعروف أن الأنموذج الذي يربط المتغير المعتمد (معدل عائد محفظة السوق) بالمتغيرات الوسيطة

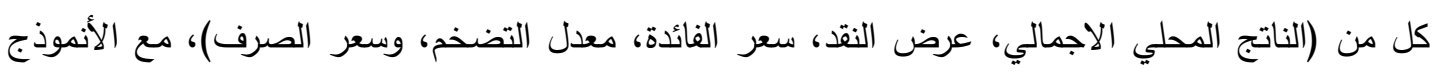
الذي يربط المتغير المستقل (معدل سعر النفط) بالمتغيرات الوسيطة فهو أنموذج انحدار خطي، وهذا يعني أن الن الني

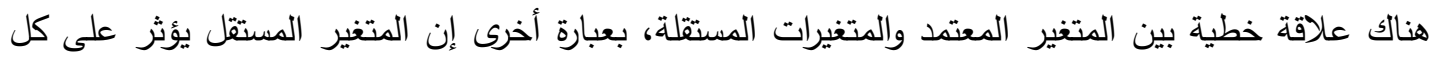

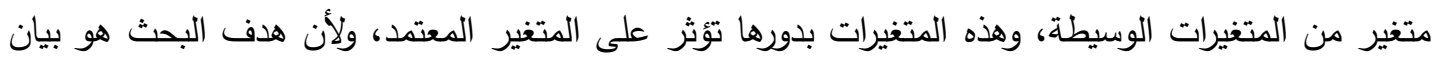

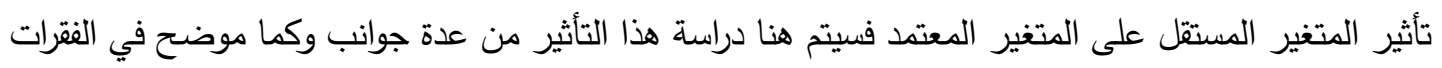
الآتية:

أ- العلاقة المباشرة بين المتغير المستقل والمتغير المعتمد: وهذا يعني أن المتغير المستقل يؤثر بشكل مباشر

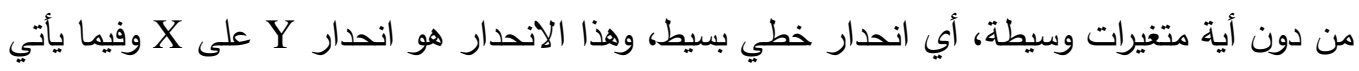
مقدرات المربعات الصغرى لمعلمات هذه المعادلة:

$Y_{t}=\delta_{0}+\delta_{1} X_{1 t}+\omega_{t \ldots \ldots}$

هذه المعادلة هي التي تعرف بمعادلة انحدار (Y) على (X)، والجدول ( ) تشير إلى نتائج مقدرات المربعات الصغرى لمعلمات المعادلة.

جدول (1) تقدير معلمات أنموذج الانحدار لمتغير Y (X (X)

\begin{tabular}{|c|c|c|c|c|}
\hline Prob. & t-Statistic & Coefficient & المتغير المستقل & $ت$ \\
\hline 0.4146 & 0.827241 & $\cdot, Y) \leq 707$ & الثابت & .1 \\
\hline 0.0298 & -2.281481 & -0.044544 & $X$ &.$r$ \\
\hline
\end{tabular}

المصدر: من إعداد الباحث بالاعتماد على بيانات البحث ومخرجات برنامج Eviews-10.

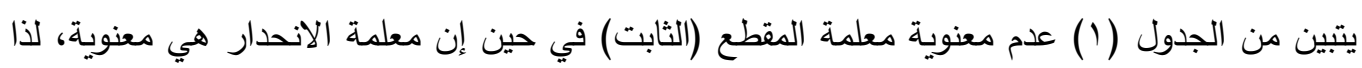
سيتم فقط تقدير معلمة الانحدار في الجدول (Y) وباستبعاد المقطع الحد (الثابت) وكما يأتي: 
جدول (Y) تقدير معلمات أنموذج الانحدار لمتفير (X)

\begin{tabular}{|c|c|c|c|c|c|}
\hline Adjusted $\mathrm{R}^{2}$ & $\mathrm{R}^{2}$ & Prob. & $\mathrm{t}$-Statistic & Coefficient & المستغلير \\
\hline $12.8 \%$ & $12.8 \%$ & 0.0256 & -2.344285 & -0.045463 & $x$ \\
\hline
\end{tabular}

المصدر: من اعداد الباحث بالاعتماد على بيانات البحث ومخرجات برنامج Eviews-10.

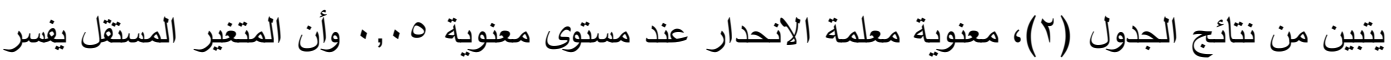

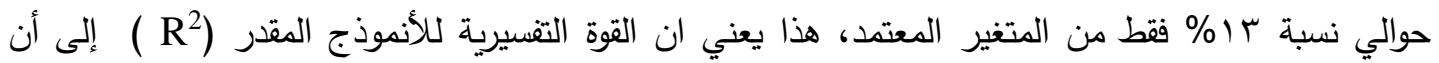
التغيرات الحاصلة في المتغير التابع تفسر بواسطة التغيرات في المتغيرات المستقلة، وبما ان المتغير المستهدف هو المتغير المعتمد، فنسبة التأثيرات لمتغيرات المستقلة على المتغير المعتمدة كلما تكون عالية تكون افضل،

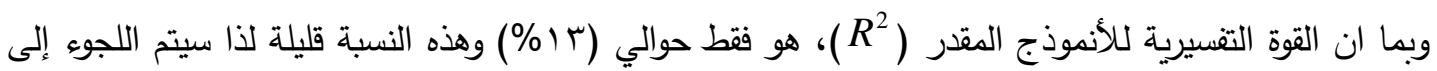
نماذج أخرى مثل نماذج العلاقة المبنية على متغيرات وسيطة بغية الحصول على نسبة افضل الفول. ب- العلاقة المبنية على متغيرات وسيطة: وهذا يعني أن المتغير المستقل يؤثر على متغيرات وسيطة وهذه بغنه

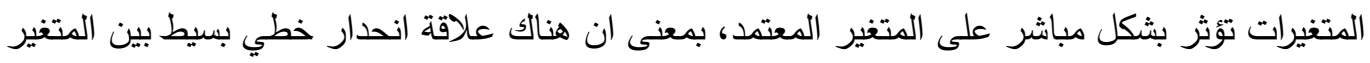
المستقل وكل متغير من المتغيرات الوسيطة، ومعادلة انحدار خطي متعدد تربط بين المتغيرات الوسيطة لتصني والمتغير المعتمد، والجدول (r) يمثل تقدير الأنموذج الخاص بكل متغيرنير وسيط بالنسبة للمتغير المستقل:

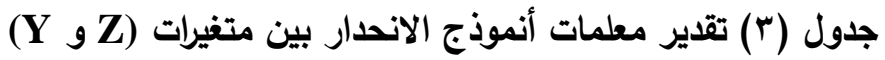

\begin{tabular}{|c|c|c|c|c|c|}
\hline .Prob & $\begin{array}{l}\mathrm{t}- \\
\text { Statistic }\end{array}$ & Coefficient & يط (المعتمد) & & \\
\hline 0.3292 & 0.991958 & 0.014561 & $\alpha$ & \multirow[t]{2}{*}{ Z1 } & 1. \\
\hline 0.0001 & 4.615226 & 0.005097 & $\beta$ & & \\
\hline 0.8821 & -0.149669 & -0.003331 & $\alpha$ & \multirow[t]{2}{*}{$\mathrm{Z} 2$} & 2. \\
\hline 0.0001 & -4.731222 & -0.007855 & $\beta$ & & \\
\hline 0.0000 & 15.81426 & 4.516786 & $\alpha$ & \multirow[t]{2}{*}{$\mathrm{Z3}$} & 3. \\
\hline 0.3661 & 0.917707 & 0.019722 & $\beta$ & & \\
\hline 0.0002 & 4.184482 & 1.546641 & $\alpha$ & \multirow[t]{2}{*}{$\mathrm{Z4}$} & 4. \\
\hline 0.7987 & -0.257342 & -0.007157 & $\beta$ & & \\
\hline 0.8963 & -0.131497 & -0.000645 & $\alpha$ & \multirow[t]{2}{*}{$\mathrm{Z5}$} & 5. \\
\hline 0.5701 & 0.574377 & 0.000210 & $\beta$ & & \\
\hline
\end{tabular}

المصدر: من اعداد الباحث بالاعتماد على بيانات البحث ومخرجات برنامج Eviews-10.

يلاحظ من نتائج الجدول (r) عن عدم معنوية الثوابت في كل من معادلة (Z) (Z) بمتغيرات كل من (الناتج المحلي الاجمالي، عرض النقد، وسعر الصرف)، لذا سيتم استخرج الثوابت من هذه منه المعادلات، في حين ان الثوابت معنوية في كل من (Z Z Z Z Z)، المعبرة عن متغيرين لكل من (سعر الفائدة ومعدل التضخم)، ولكن معاملات الانحدار غير معنوية، لذا سيتم اللجوء إلى استخراج هذه الثوابت أيضاً فلا يمكن الاستفادة منها في التحليل في حالة عدم معنوية معاملات الانحدار ، وفي الجدول (ع) سيتم تقدير المعلمات بعد استبعاد جميع الثوابت وكما يأتي:

TANMIYAT AL-RAFIDAIN (P-ISSN: 1609-591X; E-ISSN: 2664-276X) تنمية الرافدين 
جدول (ع ) تقدير معلمات أنموذج الانحدار بين متغيرات (Zو Y Y ) بعد استبعاد الثوابت

\begin{tabular}{|c|c|c|c|c|c|c|}
\hline Adjusted $\mathrm{R}^{2}$ & $\overline{\mathrm{R}^{2}}$ & Prob. & t-Statistic & Coefficient & المتغير المعتمد & ت \\
\hline $39.6 \%$ & $39.6 \%$ & 0.0001 & 4.567370 & 0.005035 & $\mathrm{Z1}$ & 1. \\
\hline $43.5 \%$ & $43.5 \%$ & 0.0000 & -4.813512 & -0.007835 & $\mathrm{Z2}$ & 2. \\
\hline- & - & 0.9952 & 0.006014 & 0.000388 & $\mathrm{Z3}$ & 3. \\
\hline- & - & 0.6913 & -0.400813 & -0.013777 & $\mathrm{Z4}$ & 4. \\
\hline- & - & 0.5552 & 0.596639 & 0.000214 & $\mathrm{Z5}$ & 5. \\
\hline
\end{tabular}

المصدر: من اعداد الباحث بالاعتماد على بيانات البحث ومخرجات برنامج Eviews-10.

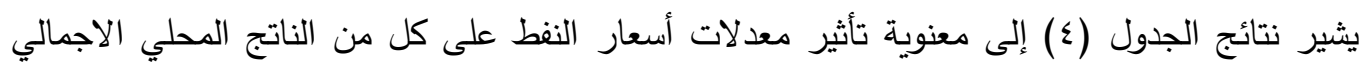

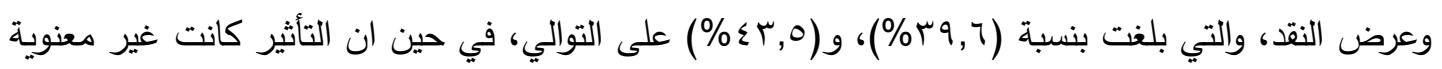

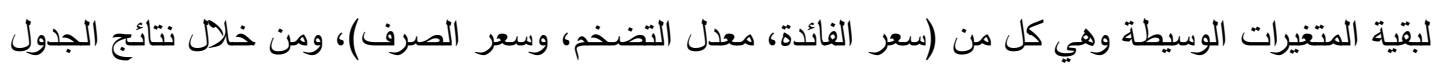
(ع) يمكن التأكد منها عبر ما سيعرض في الجدول (0)، للوصول إلى مدى معنوية تأثير المتغيرات الوسيطة التهن على المتغير المعتمد، إلى ذلك سيتم اختيار المتغيرات باستخدام طريقة الاختيار الأمامي.

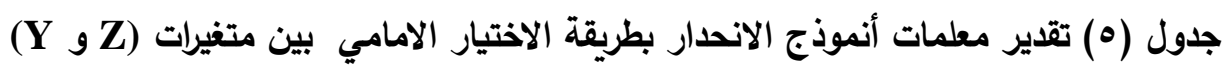

\begin{tabular}{|c|c|c|c|c|c|}
\hline Adjusted $\mathrm{R}^{2}$ & $\mathrm{R}^{2}$ & Prob. & t-Statistic & Coefficient & لمتغير المستقل \\
\hline $34.1 \%$ & $34.1 \%$ & 0.0003 & 4.100152 & 5.958351 & $\mathrm{Z}_{2}$ \\
\hline
\end{tabular}

المصدر: من اعداد الباحث بالاعتماد على بيانات البحث ومخرجات برنامج Eviews-10.

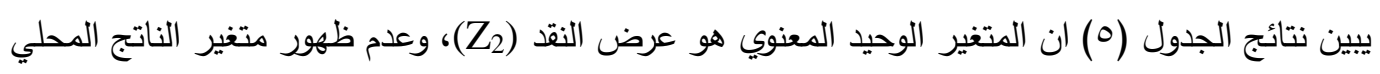

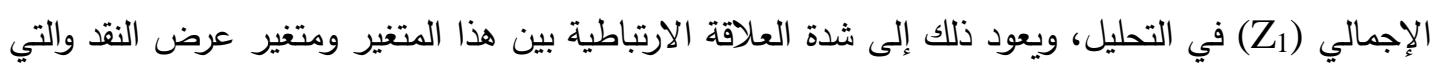

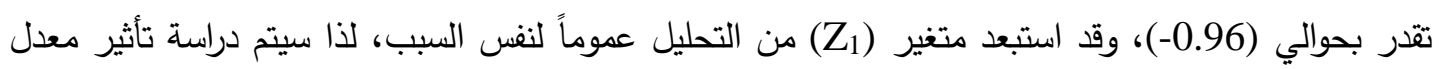

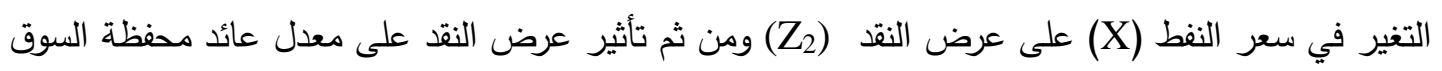

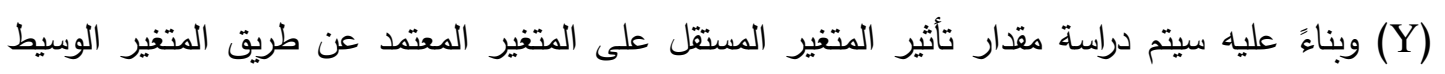

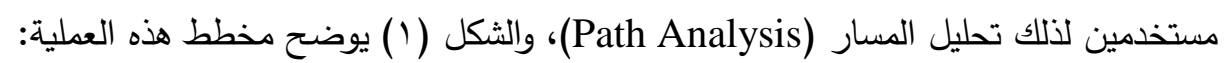

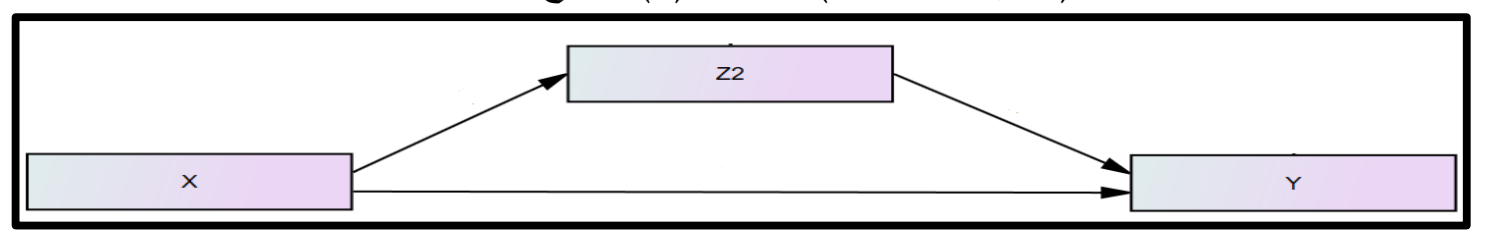

شكل (1): مخطط يوضح تحليل المسار لأثر (X على Y) بواسطة (Z) المصدر: المخطط من إعداد الباحث بالاعتماد على نتائج مخرجات الجداول السابقة. وتظهر نتائج تحليل المسار في الجدول (†)، فيما يتعلق بالتأثيرات الكلية المباشرة وغير المباشرة وكذلك معامل التحديد بين متغيرات الدراسة: 
جدول (†) التأثيرات الكلية المباشرة وغير المباشرة بين متغيرات البحث

\begin{tabular}{|c|c|c|c|}
\hline $\mathbf{R}^{2}$ & Coefficient & التأثيرات & ت \\
\hline $14.67 \%$ & -0.383 & التأثيرات الكلية & .1 \\
\hline $0.07 \%$ & 0.026 & التأثيرات المباشرة &.$r$ \\
\hline $14.60 \%$ & -0.409 & التأثيرات غير المباشرة & r. \\
\hline
\end{tabular}

المصدر: من اعداد الباحث بالاعتماد على بيانات البحث ومخرجات برنامج Amos-26.

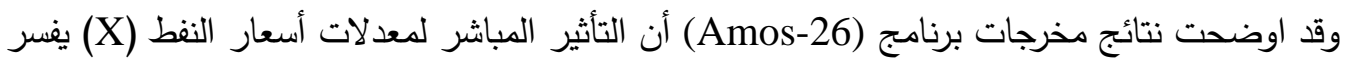
فقط حوالي (V•, ••\%) من معدل عائد محفظة السوق (Y)، بينما ظهر التأثير غير المباشر من خلال متغير

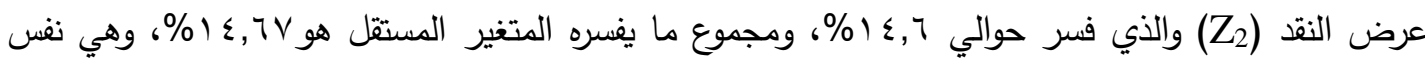
القيمة التي تم الحصول عليها في انحدار (Y) على (X)، واختلاف قيم معامل التحديد بسبب المعادلة المستخدمة

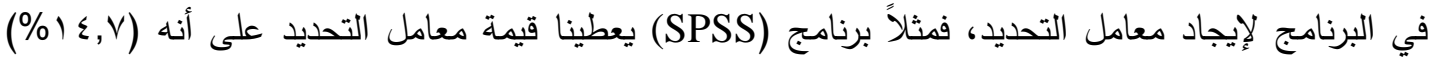

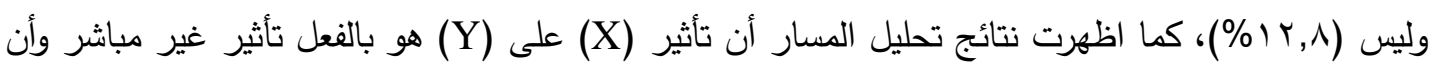

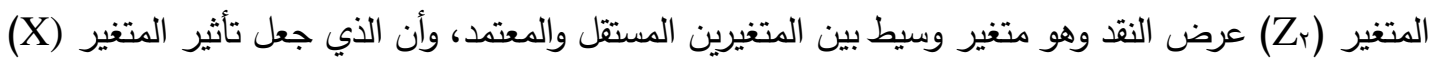

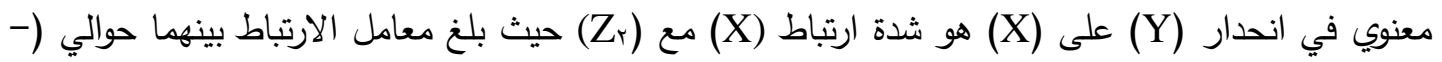

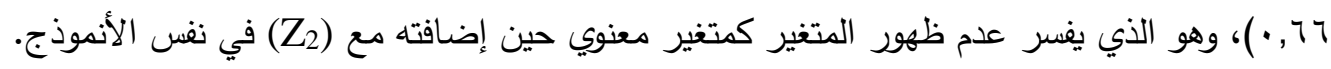
وعن محاولة استخدام تحليل الانحدار غير الخطي من خلال نماذجه الثلاثة بغية الحصول إلى نتائج تعطي

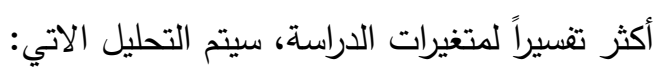
أ- انحدار متعدد حدود: اي أن المتغير المستقل ممثلة بمعدلات أسعار النفط سيؤثر على المتغير المعتمد

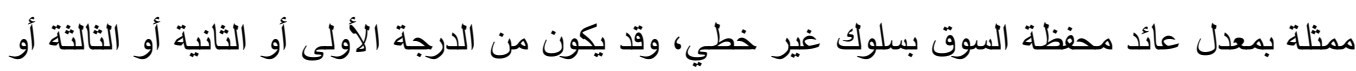

درجة أعلى، وفي الجدول (V) سيتم استخدام طريقة الاختيار الأمامي لاختيار الدرجة المناسبة.

جدول (V) نتائج أنموذج انحدار متعدد الحدود بين متغيرات البحث

\begin{tabular}{|c|c|c|c|c|c|}
\hline Adjusted R & $\mathbf{R}^{2}$ & Prob. & t-Statistic & Coefficient & المتغير| \\
\hline \multirow{3}{*}{$79.4 \%$} & \multirow{3}{*}{$81.4 \%$} & 0.0010 & 3.676209 & 0.066674 & $X$ \\
\cline { 3 - 6 } & & 0.0011 & -3.628697 & -0.004630 & $X^{2}$ \\
\cline { 3 - 6 } & & 0.0000 & -8.289790 & -0.000218 & $X^{3}$ \\
\cline { 3 - 6 } & 0.0000 & 6.197017 & 0.00000803 & $X^{4}$ \\
\hline
\end{tabular}

المصدر: من اعداد الباحث بالاعتماد على بيانات البحث ومخرجات برنامج

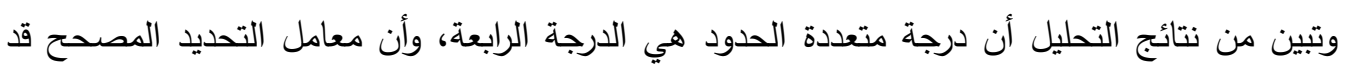
اقترب من (1 \%))، وهذا رقم كبير جداً مقارنة مع ما تم الحصول عليه في بقية النماذج السابقة. ب- الانحدار اللوغاريتمي: في هذا الأنموذج سيتم دراسة ثلاث حالات مختلفة لمتغيرات الدراسة من المتغير المستقل والمعتمد، وان الجدول (^)، يلخص نتائج هذه الحالات من خلال ما تم الحصول نلات عليه. 
جدول (^) نتائج أنموذج الانحدار اللوغاريتمي للحالات الثلاثة بين متغيرات البحث

\begin{tabular}{|c|c|c|c|c|c|c|}
\hline Prob. & t-Statistic & Coefficient & المعلمات & المتغير المستقل & المتغير & ت \\
\hline 0.9366 & 0.080203 & 0.074043 & $\alpha$ & \multirow{2}{*}{$\log (X)$} & \multirow{2}{*}{$\log (Y)$} & \multirow{2}{*}{.1} \\
\hline 0.0968 & -1.714263 & -0.456562 & $\beta$ & & & \\
\hline 0.0000 & -9.893327 & -1.492835 & $\alpha$ & \multirow{2}{*}{$X$} & \multirow{2}{*}{$\log (Y)$} & \multirow{2}{*}{.4} \\
\hline 0.6679 & -0.433345 & -0.004920 & $\beta$ & & & \\
\hline 0.0001 & 4.709646 & 6.487181 & $\alpha$ & \multirow{2}{*}{$\log (X)$} & \multirow[b]{2}{*}{$\mathrm{Y}$} & \multirow{2}{*}{ r } \\
\hline 0.0001 & -4.585659 & -1.822223 & $\beta$ & & & \\
\hline
\end{tabular}

المصدر: من اعداد الباحث بالاعتماد على بيانات البحث ومخرجات برنامج Eviews-10.

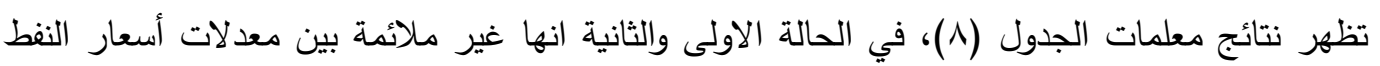

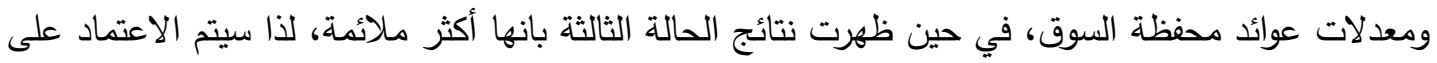
الاخيرة، ونتائج الجدول التالي يوضح ذلك:

جدول (9) نتائج الانحدار اللوغاريتمي بانحدار (Y) على (X)

\begin{tabular}{|c|c|c|c|c|c|}
\hline Adjusted $\mathbf{R}^{2}$ & $\mathbf{R}^{2}$ & Prob. & t-Statistic & Coefficient & المتغير المستقل \\
\hline \multirow{2}{*}{$39.2 \%$} & \multirow{2}{*}{$41.2 \%$} & 0.0001 & 4.709646 & 6.487181 & الثابت \\
\hline & & 0.0001 & -4.585659 & -1.822223 & $\log (X)$ \\
\hline
\end{tabular}

المصدر: من اعداد الباحث بالاعتماد على بيانات البحث ومخرجات برنامج Eviews-10.

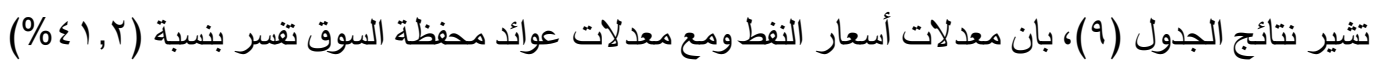
من التغيرات الحاصلة في معدل عوائد محفظة السوق.

ت- الانحدار الجيبي: في هذا الأنموذج ايضا سيتم دراسة ثلاثة حالات ولكن بأشكال مختلفة، والجدول (· ( ) يلخص هذه الحالات من خلال المحالات التي تم الحصول عليها. جدول (• 1) نتائج أنموذج الانحدار الجيبي للحالات الثلاثة بين متغيرات البحث

\begin{tabular}{|c|c|c|c|c|c|c|}
\hline .Prob & t-Statistic & Coefficient & المعلمات & المتغتير & المتغير & ت \\
\hline 0.7813 & -0.280065 & -0.007043 & $\alpha$ & \multirow[t]{2}{*}{$\operatorname{Sin}(X)$} & \multirow[t]{2}{*}{$\operatorname{Sin}(Y)$} & \multirow[t]{2}{*}{1.} \\
\hline 0.3936 & 0.865572 & 0.031670 & $\beta$ & & & \\
\hline 0.7432 & -0.330706 & -0.008406 & $\alpha$ & \multirow[t]{2}{*}{$\mathrm{X}$} & \multirow[t]{2}{*}{$\operatorname{Sin}(Y)$} & \multirow[t]{2}{*}{2.} \\
\hline 0.7002 & -0.388792 & -0.000744 & $\beta$ & & & \\
\hline 0.3624 & 0.924935 & 0.256818 & $\alpha$ & \multirow[t]{2}{*}{$\operatorname{Sin}(X)$} & \multirow[t]{2}{*}{$Y$} & \multirow[t]{2}{*}{3.} \\
\hline 0.4131 & 0.829991 & 0.335305 & $\beta$ & & & \\
\hline
\end{tabular}

المصدر: من إعداد الباحثين بالاعتماد على بيانات البحث ومخرجات برنامج Eviews-10. 
إذ يلاحظ انه عند استبعاد معلمات الثوابت من الحالات الثلاث في الجدول ( • () فالنتائج بقيت غير ملائمة،

لذا لا يمكن بناء أنموذج جيبي مناسب بين معدلات أسعار النفط مع معدلات عوائد محفظة السوق.

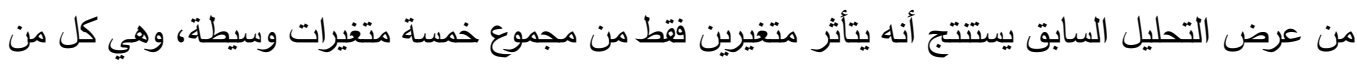
الناتج المحلي الإجمالي وعرض النقد بمعدلات تقلب أسعار النفط، وهناك علاقة غير مباشرة بين معدلات أسعار

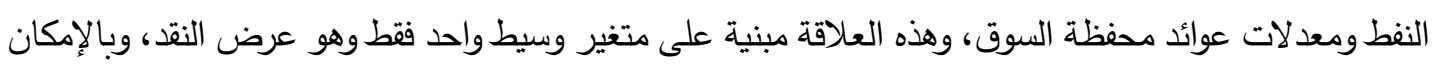

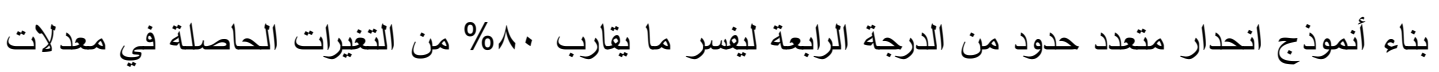

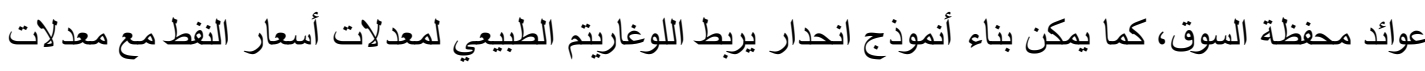

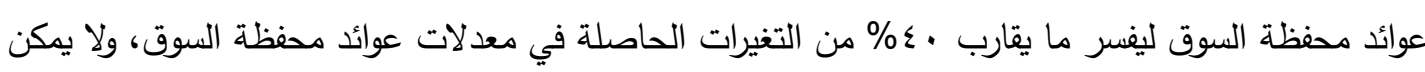
بناء أنموذج انحدار جيبي بين تلك المتغيرات. r-r. آثار تقلبات أسعار النفط على عوائد محفظة القطاعات الاقتصادية

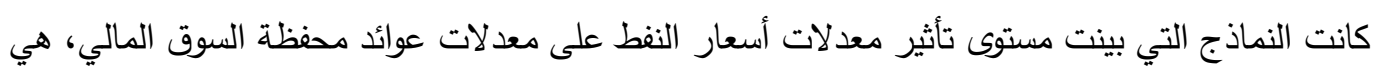
تلك النماذج المعنوية التي تم الحصول عليها كما هي موضحة في الجدول (1')، وكما يأتي:

جدول (1) (1) ترشيح الأنموذج الأكثر ملاءمة بين متغيرات البحث

\begin{tabular}{|c|c|c|}
\hline Adjusted $\mathbf{R}^{2} \%$ & الأنموذج & 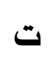 \\
\hline$M, \Lambda$ & الأنموذج الخطي البسيط & .1 \\
\hline$\vee q, \varepsilon$ & الأنموذج متعدد الحدود &.$r$ \\
\hline$r q, r$ & الأنموذج اللوغاريتمي Y على (X) & r \\
\hline
\end{tabular}

المصدر: من إعداد الباحثين بالاعتماد على نتائج النماذج السابقة.

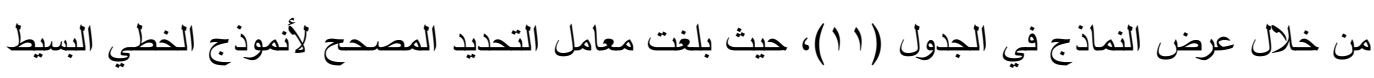

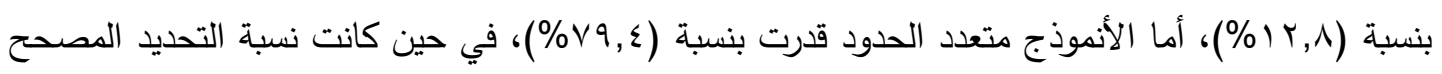

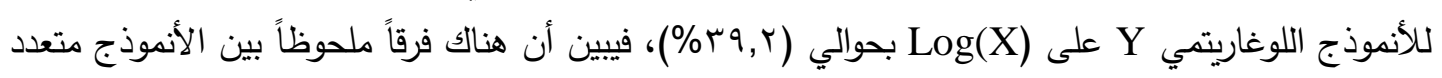

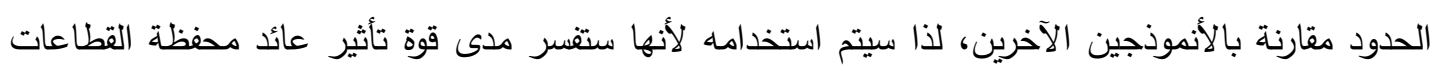
الاقتصادية للسوق المالي بتقلبات أسعار النفط، وصيغته الرياضية هي على لالى النحو الآتي: $Y_{t}=\beta_{1} X_{t}+\beta_{2} X_{t}^{2}+\beta_{3} X_{t}^{3}+\beta_{4} X_{t}^{4}+\varepsilon_{t}$

بناءً على اختيار الأنموذج متعدد الحدود فسيتم تطبيقه على القطاعات الاقتصادية لسوق العراق للأوراق

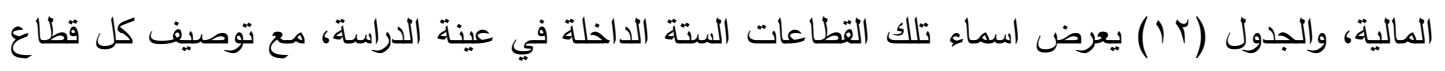
برمز معين لسهولة احتسابه في العمليات التحليلية. 
جدول (r T) القطاعات الاقتصادية لسوق العراق للأولق المالية

\begin{tabular}{|c|c|c|}
\hline الرمز & القطاعات & ت \\
\hline $\mathrm{Y}_{1}$ & المصرفي & .1 \\
\hline $\mathrm{Y}_{2}$ & التأمين &.$r$ \\
\hline $\mathrm{Y}_{3}$ & الخدمات & r \\
\hline $\mathrm{Y}_{4}$ & الفنادق والسياحة &.$\varepsilon$ \\
\hline $\mathrm{Y}_{5}$ & الصناعة & .0 \\
\hline $\mathrm{Y}_{6}$ & الزراعي & .7 \\
\hline
\end{tabular}

الجدول من إعداد الباحثين.

والان بعد ترشيح الأنموذج الأكثر ملاءمة، فسيتم تطبيقه وبشكل منفصل على جميع القطاعات الاقتصادية،

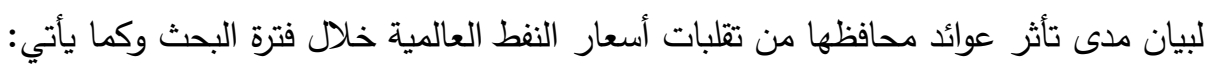

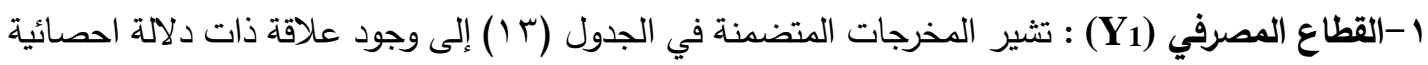
بين عائد محفظة القطاع المصرفي وتقلبات أسعار النفط، حيث تبين أن جميع القيم الاحتمالية هي معنوية لأنها أقل من (0\%)، وبذلك نرفض فرضية العدم ونقبل بالفرضية البديلة، كما وفسرت نسبة معامل التحديد المصحح

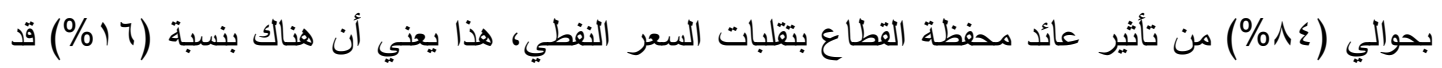

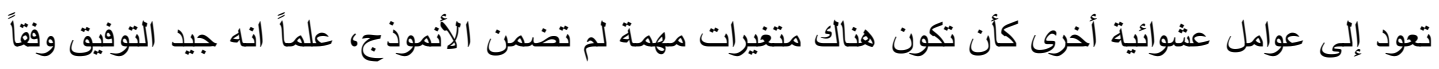
لقيمة معامل التحديد المصحح. جدول (r I ) تأثير تقلبات أسعار النفط على عوائد محفظة القطاع المصرفي

\begin{tabular}{|c|c|c|c|c|c|}
\hline Adjusted R & $\mathrm{R}^{2}$ & Prob. & t-Statistic & Coefficient & المتغير| \\
\hline \multirow{3}{*}{$83.78 \%$} & \multirow{3}{*}{$85.35 \%$} & 0.0011 & 3.651678 & 0.124835 & $X$ \\
\cline { 3 - 7 } & & 0.0000 & -6.081049 & -0.014625 & $X^{2}$ \\
\cline { 3 - 6 } & & 0.0000 & -9.487688 & -0.000471 & $X^{3}$ \\
\cline { 3 - 6 } & 0.0000 & 8.671028 & 0.0000212 & $X^{4}$ \\
\hline
\end{tabular}

المصدر: من إعداد الباحثين بالاعتماد على بيانات البحث ومخرجات برنامج r- قطاع التأمين (Y2): الجدول (乏) () يعرض نتائج علاقة الارتباط بين عائد محفظة قطاع التأمين العراقي وتقلبات أسعار النفط، ويفسر عدم وجود أي علاقة بين هذين المتغيرين، والقيم الاحتمالية هي غير مانير معنوية، لأن أغلبها أكبر من (0\%) وبنسب عالية، هذا ما يدعم قبول فرضية العدم ورفض الفرضية البديلة. 
جدول (ع ا ) تأثير تقلبات أسعار النفط على عوائد محفظة قطاع التأمين

\begin{tabular}{|c|c|c|c|c|c|}
\hline Adjusted $\mathrm{R}^{2}$ & $\mathrm{R}^{2}$ & Prob. & $\mathrm{t}$-Statistic & Coefficient & المتغير المستقل \\
\hline \multirow{4}{*}{$00.00 \%$} & \multirow{4}{*}{$00.00 \%$} & 0.9546 & 0.057404 & 0.003695 & $\mathrm{X}$ \\
\hline & & 0.3145 & -1.024296 & -0.004639 & $\mathrm{X}^{2}$ \\
\hline & & 0.7597 & -0.308814 & -0.0000289 & $\mathrm{X}^{3}$ \\
\hline & & 0.2449 & 1.187821 & 0.00000546 & $X^{4}$ \\
\hline
\end{tabular}

المصدر: من إعداد الباحثين بالاعتماد على بيانات البحث ومخرجات برنامج هذا يشير بأن لتقبات أسعار النفط ليس له اي علاقة ارتباط قوية مع عوائد محفظة قطاع التأمين العراقي

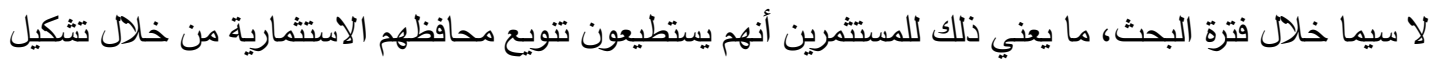
عوائد قطاعات اقتصادية ذات علاقة تأثير ضعيفة او منعدمة بتقلبات أسعار النفط. r- قطاع الخدمات (Y3)): من خلال عرض نتائج الجدول (10)، تبينت أن معامل التحديد المصحح بين

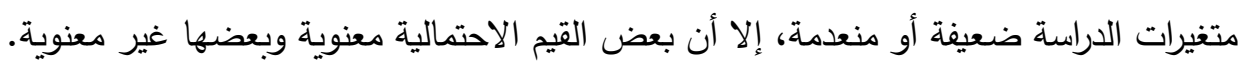
جدول (0 1 ) تأثير تقلبات أسعار النفط على عوائد محفظة قطاع الخدمات

\begin{tabular}{|c|c|c|c|c|c|}
\hline Adjusted $\mathbf{R}^{2}$ & $\mathbf{R}^{2}$ & Prob. & t-Statistic & Coefficient & المتغير المستقل \\
\hline \multirow{4}{*}{$00.00 \%$} & \multirow{4}{*}{$00.00 \%$} & 0.6634 & 0.439860 & 0.003695 & $\mathrm{X}$ \\
\hline & & 0.0401 & -2.152501 & -0.004639 & $\mathrm{X}^{2}$ \\
\hline & & 0.3165 & -1.019867 & -0.0000289 & $\mathrm{X}^{3}$ \\
\hline & & 0.0482 & 2.065862 & 0.00000546 & $\mathrm{X}^{4}$ \\
\hline
\end{tabular}

المصدر: من إعداد الباحثين بالاعتماد على بيانات البحث ومخرجات برنامج

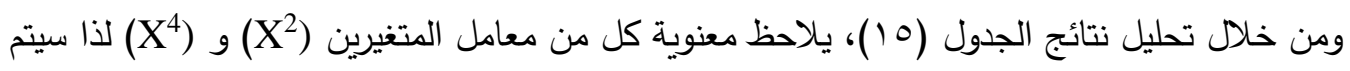
تكوين الأنموذج فقط بدلالتهما، وكما هو موضح في مخرجات الجدول (7 (1): جدول (1 1 ) تأثير تقلبات أسعار النفط على عوائد محفظة قطاع الخدمات لمتفيرات (X) X

\begin{tabular}{|c|c|c|c|c|c|}
\hline Adjusted R & $\mathbf{R}^{2}$ & Prob. & t-Statistic & Coefficient & المتغير \\
\hline \multirow{2}{*}{$00.00 \%$} & \multirow{2}{*}{$00.00 \%$} & 0.0753 & -1.842272 & -0.002378 & $X^{2}$ \\
\cline { 3 - 7 } & & 0.0914 & 1.743719 & 0.00000208 & $X^{4}$ \\
\hline
\end{tabular}

المصدر: من إعداد الباحثن بالاعتماد على بيانات البحث ومخرجات برنامج Eviews-10.

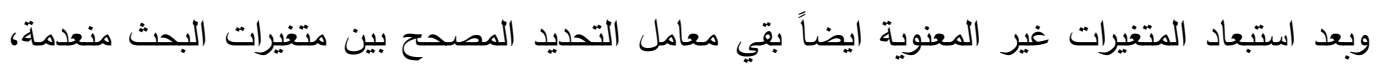

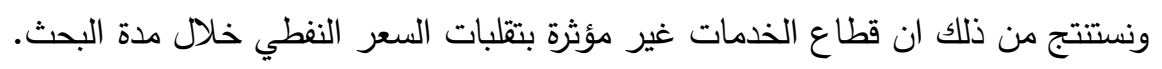

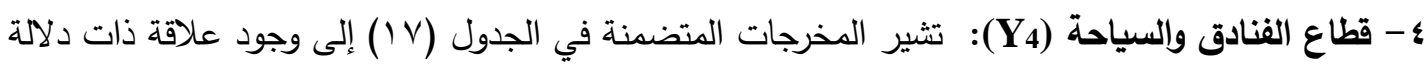
احصائية بين عائد محفظة قطاع الفنادق والسياحة العراقي وتقلبات أسعار النفط، حيث بلغ الغ معامل التحديد

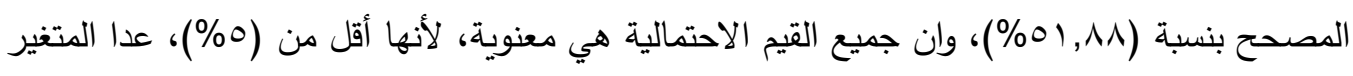

$$
\text { (X }
$$

TANMIYAT AL-RAFIDAIN (P-ISSN: 1609-591X; E-ISSN: 2664-276X) تنمية الرافدين 
جدول (IV) تأثير تقلبات أسعار النفط على عوائد محفظة قطاع الفنادق والسياحة

\begin{tabular}{|c|c|c|c|c|c|}
\hline Adjusted $\mathbf{R}^{2}$ & $\mathbf{R}^{2}$ & Prob. & t-Statistic & Coefficient & المتغير المستقل \\
\hline \multirow{4}{*}{$51.88 \%$} & \multirow{4}{*}{$56.53 \%$} & 0.0268 & 2.337294 & 0.042786 & $\mathrm{X}$ \\
\hline & & 0.0583 & -1.974232 & -0.002543 & $\mathrm{X}^{2}$ \\
\hline & & 0.0001 & -4.706472 & -0.000125 & $\mathrm{X}^{3}$ \\
\hline & & 0.0023 & 3.350713 & 0.00000438 & $\mathrm{X}^{4}$ \\
\hline
\end{tabular}

المصدر: من إعداد الباحثين بالاعتماد على بيانات البحث ومخرجات برنامج

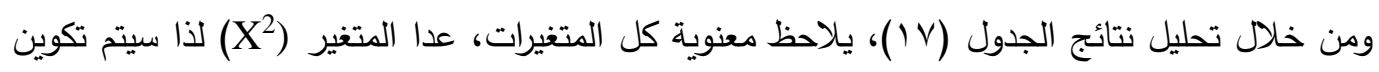
أنموذج باستبعاده، وكما هو موضح في مخرجات الجدول (1) (1).

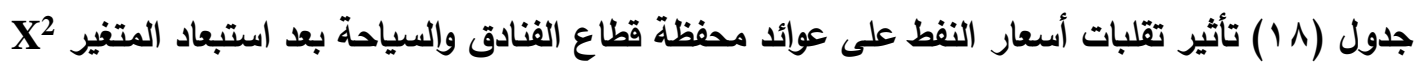

\begin{tabular}{|c|c|c|c|c|c|}
\hline Adjusted $\mathrm{R}^{2}$ & $\mathrm{R}^{2}$ & Prob. & $\mathrm{t}$-Statistic & Coefficient & المتغير المستقل \\
\hline \multirow{3}{*}{$\% \cdot v . \varepsilon v$} & \multirow{3}{*}{$\% \leq 9 . \cdot 5$} & 0.0478 & 2.066687 & 0.039514 & $X$ \\
\hline & & 0.0003 & -4.075327 & -0.000102 & $\mathrm{X}^{3}$ \\
\hline & & 0.0001 & 4.580955 & 0.00000192 & $\mathrm{X}^{4}$ \\
\hline
\end{tabular}

المصدر: من إعداد الباحثين بالاعتماد على بيانات البحث ومخرجات برنامج Eviews-10.

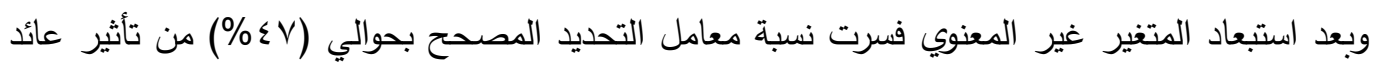

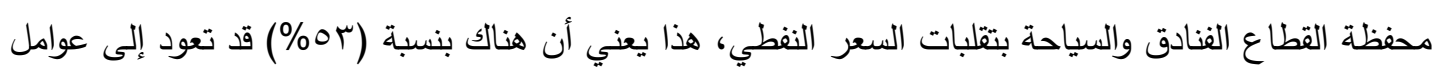

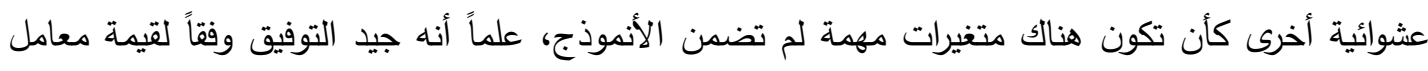
التحديد المصحح. ه- قطاع الصناعة (Y5): من خلال عرض نتائج تحليل الجدول (9 ( ) تبين أن جميع القيم الاحتمالية معنوية،

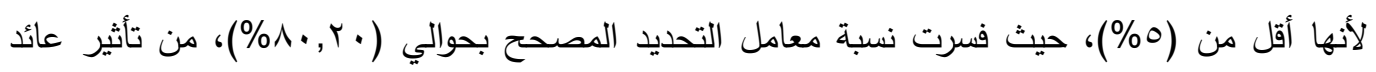

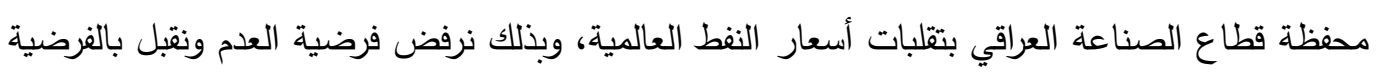

$$
\text { البديلة. }
$$

جدول (9 1 ) تأثير تقلبات أسعار النفط على عوائد محفظة قطاع الصناعة

\begin{tabular}{|c|c|c|c|c|c|}
\hline Adjusted R & $\mathrm{R}^{2}$ & Prob. & t-Statistic & Coefficient & المتئyy \\
\hline \multirow{3}{*}{$80.20 \%$} & \multirow{3}{*}{$82.12 \%$} & 0.0008 & 3.773468 & 0.170438 & $\mathrm{X}$ \\
\cline { 3 - 7 } & & 0.0004 & -4.005735 & -0.012729 & $\mathrm{X}^{2}$ \\
\cline { 3 - 7 } & & 0.0000 & -8.514007 & -0.000559 & $\mathrm{X}^{3}$ \\
\cline { 3 - 7 } & 0.0000 & 6.568100 & 0.0000212 & $\mathrm{X}^{4}$ \\
\hline
\end{tabular}

المصدر: من إعداد الباحثين بالاعتماد على بيانات البحث ومخرجات برنامج

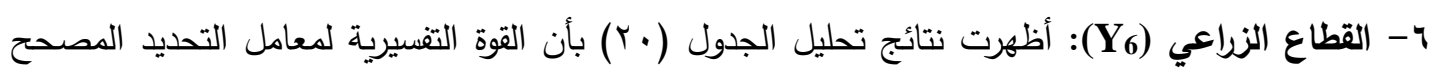
الخاص بالقطاع الزراعي العراقي، كانت بحوالي نسبة (r؟ \%) من التغيرات الحاصلة في تقلبات أسعار

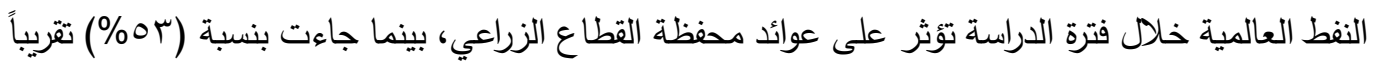


من التغيرات الحاصلة في المتغير التابع المتمثلة بعوائد محفظة القطاع الزراعي، وقد يعود ذلك السبب إلى وجود متغيرات أخرى مؤثرة غير داخلة في الأنموذج لصعوبة قياسها والتي تقع ضمن اعند المتغير العشوائي.

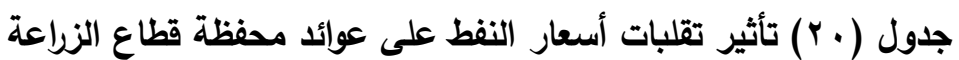

\begin{tabular}{|c|c|c|c|c|c|}
\hline Adjusted R & $\mathbf{R}^{2}$ & Prob. & t-Statistic & Coefficient & المتغير| \\
\hline \multirow{3}{*}{$42.88 \%$} & \multirow{3}{*}{$48.40 \%$} & 0.0371 & 2.189441 & 0.125838 & $X$ \\
\cline { 3 - 6 } & & 0.0085 & -2.828454 & -0.011437 & $X^{2}$ \\
\cline { 3 - 6 } & & 0.0001 & -4.566603 & -0.000381 & $X^{3}$ \\
\cline { 3 - 6 } & 0.0006 & 3.837024 & $1.58 \mathrm{E}-05$ & $X^{4}$ \\
\hline
\end{tabular}

المصدر: من إعداد الباحثين بالاعتماد على بيانات البحث ومخرجات برنامج

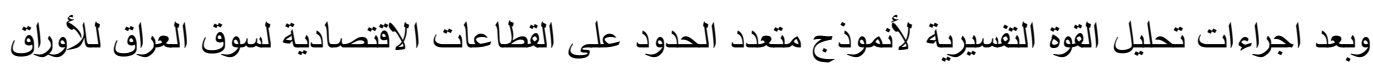
المالية، تبين أن هناك تفاوتاً بمستوى التأثير بين عوائد محفظة القطاعات من حيث تأثردها بتقلبات أسعار النفط

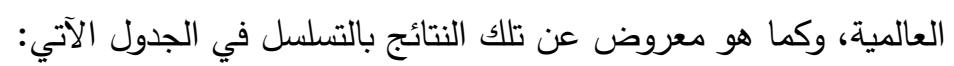

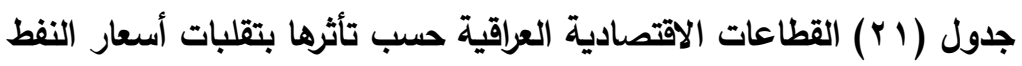

\begin{tabular}{|c|c|c|}
\hline $\begin{array}{c}\text { Adjusted R } \\
\% \\
\end{array}$ & القطاعات & ت \\
\hline$\wedge r, v \wedge$ & المصرفي & .1 \\
\hline$\Lambda \cdot, r \cdot$ & الصناعة &.$r$ \\
\hline$\varepsilon V, \cdot V$ & الفنادق والسياحة & r. \\
\hline$\varepsilon r, \wedge \wedge$ & الزراعي &.$\varepsilon$ \\
\hline$\cdots, \cdots$ & الخدمات & .0 \\
\hline$\cdots, \ldots$ & التأمين & .7 \\
\hline
\end{tabular}

الجدول من إعداد الباحث بالاعتماد على نتائج الجداول السابقة.

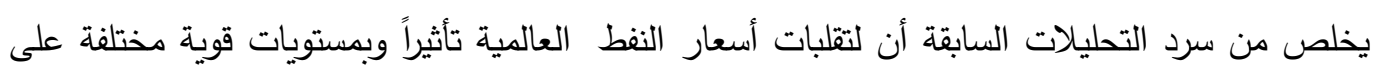

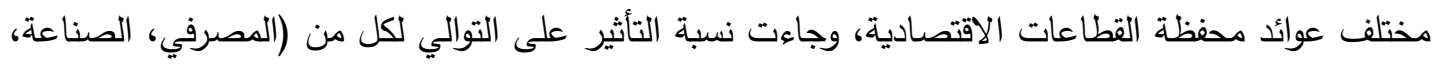

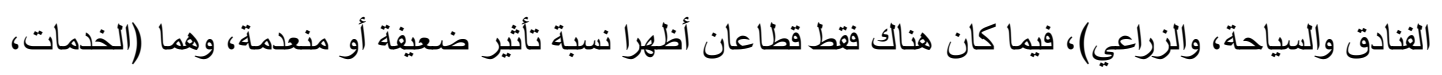
والتأمين) . ووفقنا مما سبق نقف على نفي فرضية البحث، أي انه توجد علاقة تأثير قوية وبمستويات مختلفة بين تقلبات أسعار النفط مع أغلب عوائد قطاعات محفظة سوق العراق للأوراق المالية. | الاستنتاجات ا. من بين خمسة متغيرات اقتصادية يتأثر فقط متغيران، وهما كل من الناتج المحلي الإجمالي وعرض النقد

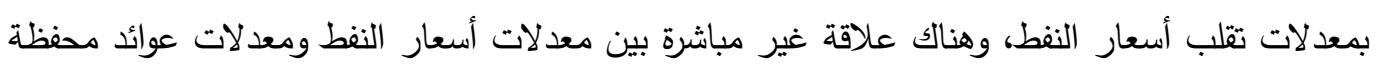
السوق، وهذه العلاقة مبنية على متغير وسيط واحد فقط وهو عرض النقد. 
r. بالإمكان بناء أنموذج انحدار متعدد حدود من الدرجة الرابعة ليفسر ما يقارب .^^\% من التغيرات الحاصلة في معدلات عوائد محفظة السوق، كما يمكن بناء أنموذج انحدار يربط اللوغاريتم الطبيعي لمعدلات السعاتهات أسعار النفط مع معدلات عوائد محفظة السوق ليفسر ما يقارب ـ؛ م من التغيرات الحاصلة في معدلات عوائد

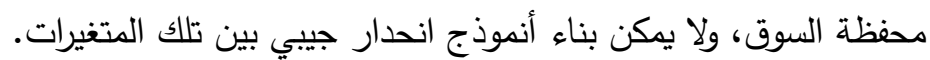
r. ت تم التوصل إلى رفض فرضية البحث والتي مفادها توجد علاقة تأثير ضعيفة بين تقلبات أسعار النفط وعوائد قطاعات محفظة السوق العراقية، لأنها ظهرت قوية وبمستويات مختلفة. ء. من بين أكثر القطاعات تأثراً بتقلبات أسعار النفط هي على التوالي (المصرفي، الصناعي، الفنادق والسياحة، والزراعي)، في حين كانت نسبة التأثير ضعيفة أو منعدمة لقطاعين فقط وهما كل من (الخدمات، والتأمين). المقترحات

ا . ضرورة فهم طبيعة تأثير تقلبات أسعار النفط وعلاقتها بعوائد أسهم محافظ الاستثمار في أسواق المال، لأن ذلك سيساعد المستثمرين للحد من المخاطر العالية.

r. بإمكان المستثر التتويع بمحفظته الاستثمارية من حيث اختياره للقطاعات الاقتصادية التي تتأثر بمستويات متفاوتة مع تغيرات أسعار النفط، لتحقيق أعلى عائد ممكن مع أقل درجة ممكنة من مستويات المخاطرة.

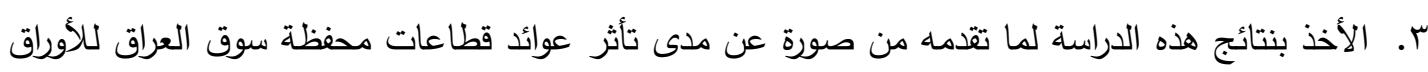
المالية بالتقلبات الاقتصادية. ع. عقد ندوات علمية وإقامة دورات تثثيفية مالية عن كيفية بناء المحافظ الاستثمارية لا سيما في الاقتصاديات

Reference

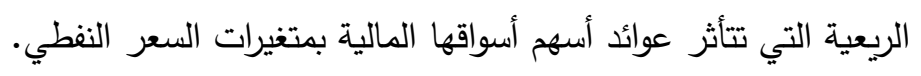

Ahmad, Muhammad Faisal Hassan Muhammad, 2019, "The Impact of Oil Price Volatility on the Performance of the Saudi Financial Market (20032016)", Journal of Economic, Administrative and Legal Sciences, Volume 3, Issue 8, National Research Center, Gaza, Palestine

Ali , Oday Salim,2017, Statistical Appendix of the Republic of Iraq, Arab Economic Journal, No 76-77, Central for Arab Unity Studies, Lebanon .

Al-Khatib, Muhammad Mahmoud, 2010, "Financial Performance and Its Impact on Share Returns in Joint-Stock Companies,", 1st Edition, Al-Hamed Publishing and Distribution House, Amman, Jordan.

Al-Mansour, Heba Allah Mustafa Al-Sayed Ali, 2018, "The Feasibility of International Diversification in Light of the Financial Crisis - An Analytical Study of a Sample of International Stock Markets", unpublished $\mathrm{PhD}$ thesis, College of Management and Economics, Karbala University, Iraq.

Al-Shakarji, Bashar Thanoon Muhammad, and Al-Taj al-Din, Mayada Salah al-Din, 2008, "The Relationship of the Stock Market Index to the Economic Status - An Analytical Study of the Riyadh Stock Exchange", Al-Rafidain Development Journal, Volume 30, Issue 89, University of Mosul, Iraq.

TANMIYAT AL-RAFIDAIN (P-ISSN: 1609-591X; E-ISSN: 2664-276X) تنمية الرافدين 
Alwan, Hussein Hassaballah, 2014, "Analyzing and measuring the relationship between oil prices and exchange rates in selected countries with special reference to Iraq", unpublished master's thesis, College of Administration and Economics, University of Baghdad, Iraq.

Aziza,S.; \& Cihan,T.; \& Symbat,N.; \& Almaz,A., 2019,"The effects of changes in oil prices on the Russian economy", Revista: espacios, Vol. (40) ,No. (14).

Benakovic, Dubravca; \& Posedel, Petra; 2010, "Do Macro Economic Factors Matter for Stock Returns? Evidence from Estimating a Multifactor Model on the Croatian Market", University of Zagreb, Faculty of Economics, Working Paper, No. (10-12).

Brown, Stephen P.; \& Yücel, Mine K.; 1999,"Oil Prices and U.S Aggregate Economic Activity :A question of Neutrality", Economic and Financial Review, Federal Reserve Bank of Dallas, Second Quarter.

Central Bank of Iraq, Annual Statistical Bulletin, Various Issues, (https://www.cbi.iq). Central Statistics organization Iraq, Various Issues, (http://cosit.gov.iq/ar).

Clare,A.; \& Thomas, S.; 2015, "Financial Market Indices: Facilitating Innovation

- Monitoring Markets", Centre For Asset Management Research, Cass Business School, London.

Iraq Stock Exchange, Annual Report, Various Issues ,(www.isxiq.net).

Ito,Katsuya, 2008, "Oil price and macro economy in Russia", Economics Bulletin, Vol. (17), No. (17).www.ideas.repec.org.

Matar, Walid; \& Al-Fattah, Saud M.; Atallah, Tarek; \& Pierru, Axel; 2013, " An introduction to oil market volatility analysis", OPEC Energy Review, Vol. (37), No. (3).

Maysami, Ramin, Cooper; \& Howe, Lee, Chuin; \& Hamza Mohamed, Atkin; 2004, "Relationship between Macroeconomic variables and Stock Market Indices: Cointegration Evidence from Stock Exchange of Singapore AllS Sector Indices", Journal Pengurusan, Vol. (24).

Mustafa, K.; \& Ahmed, R.; \& Siddiqui, A., 2013, "Money Supply and Equity Price Movements in Pakistan", European Journal of Business and Management, Vol. (5), No. (5).

Rentschler ,Jun E. , 2013, "Oil price v olatility , Economic growth and the hedging role of renewable energy", The world bank, Sustainable development network, Office of the chief economist.

The Arab Planning Institute, 2004, "Financial Market Analysis", a periodical series dealing with development issues in the Arab countries, Kuwait, Year 3, Issue 27.

World Bank, Various Issues ,Data on web site: (http://publication.worldbank.org./WDI/indicators).

Yara, Samir Abdel-Saheb, 2016, "Choosing the Optimal Investment Portfolio - An Applied Study Proposal Form", unpublished PhD thesis, College of Administration and Economics, Al-Mustansiriya University, Iraq.

TANMIYAT AL-RAFIDAIN (P-ISSN: 1609-591X; E-ISSN: 2664-276X) تنمية الرافدين 